\title{
Search for electroweak production of charginos and neutralinos using leptonic final states in pp collisions at $\sqrt{s}=7 \mathrm{TeV}$
}

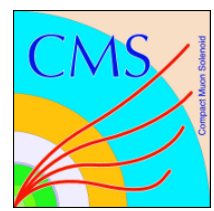

\section{The CMS collaboration}

E-mail: cms-publication-committee-chair@cern.ch

ABstract: The 2011 dataset of the CMS experiment, consisting of an integrated luminosity of $4.98 \mathrm{fb}^{-1}$ of pp collisions at $\sqrt{s}=7 \mathrm{TeV}$, enables expanded searches for direct electroweak pair production of charginos and neutralinos in supersymmetric models as well as their analogs in other models of new physics. Searches sensitive to such processes, with decays to final states that contain two or more leptons, are presented. Final states with three leptons, with a same-sign lepton pair, and with an opposite-sign lepton pair in conjunction with two jets, are examined. No excesses above the standard model expectations are observed. The results are used in conjunction with previous results on four-lepton final states to exclude a range of chargino and neutralino masses from approximately 200 to $500 \mathrm{GeV}$ in the context of models that assume large branching fractions of charginos and neutralinos to leptons and vector bosons.

KEYWORDS: Hadron-Hadron Scattering 


\section{Contents}

1 Introduction 1

2 Detector, online selection, and object selection 5

$\begin{array}{lll}3 & \text { Searches in the three-lepton final state } & 6\end{array}$

$\begin{array}{lll}3.1 & \text { Searches with three leptons using } E_{\mathrm{T}}^{\text {miss }} \text { shape } & 7\end{array}$

3.2 Searches with three leptons using $M_{\ell \ell}$ and $M_{\mathrm{T}} \quad 7$

3.2.1 Background due to WZ production 8

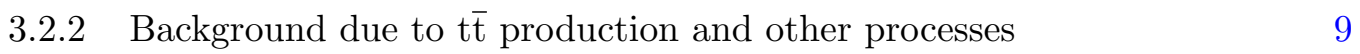

3.2.3 Observations in the three-lepton search with $M_{\ell \ell}$ and $M_{\mathrm{T}} \quad 10$

4 Searches in the same-sign two-lepton final state 10

5 Searches in the $\mathrm{WZ} / \mathrm{ZZ}+E_{\mathrm{T}}^{\text {miss }}$ final state with two leptons and two jets 14

6 Interpretations of the searches $\quad \mathbf{1 5}$

6.1 Limits on SMS from the search with three leptons using $E_{\mathrm{T}}^{\mathrm{miss}}$ shape $\quad 16$

6.2 Limits on SMS from the search with three leptons, $M_{\ell \ell}$, and $M_{\mathrm{T}}$, and from $\begin{array}{ll}\text { same-sign dilepton searches } & 17\end{array}$

6.3 Limits on SMS and GMSB from the $\mathrm{WZ} / \mathrm{ZZ}+E_{\mathrm{T}}^{\text {miss }}$ final state with two or more leptons

6.3.1 Limits on SMS with on-shell $\mathrm{W}$ and $\mathrm{Z}$ from $\mathrm{WZ}+E_{\mathrm{T}}^{\mathrm{miss}}$ and threelepton analyses 20

6.3.2 Limits on a Z-enriched GMSB model from $\mathrm{ZZ}+E_{\mathrm{T}}^{\mathrm{miss}}$ and four-lepton search 20

6.4 Summary of excluded masses for chargino-neutralino pair production 22

$\begin{array}{llr}7 & \text { Summary } & 22\end{array}$

A Signal efficiency model for the three-lepton analysis with $M_{\ell \ell}$ and $M_{\mathrm{T}} \quad 25$

$\begin{array}{ll}\text { The CMS collaboration } & 30\end{array}$

\section{Introduction}

Many searches for physics beyond the standard model (BSM) performed by experiments at the CERN Large Hadron Collider (LHC) have focused on models with cross sections dominated by the production of new heavy strongly interacting particles, with final states characterized by large hadronic activity. These searches are well justified since strongly interacting particles can be produced with large cross sections and hence be observable with 
early LHC data. In the context of supersymmetry (SUSY) [1-7], such models lead mainly to the production of the strongly interacting squarks and gluinos, the SUSY partners of the quarks and gluons. In contrast, in this paper we describe searches for BSM physics dominated by the direct electroweak production of particles that might not yield large hadronic activity, and that may therefore have eluded detection in early searches. This signature characterizes SUSY models with pair-production of electroweak charginos $\widetilde{\chi}^{ \pm}$and neutralinos $\widetilde{\chi}^{0}$, mixtures of the SUSY partners of the gauge bosons and Higgs bosons. Depending on the mass spectrum, the charginos and neutralinos can have significant decay branching fractions $(\mathrm{BF})$ to leptons or vector bosons, resulting in final states that contain either onshell vector bosons or three-lepton states with continuous pair-mass distributions [8-13]. In either case, neutrino(s) and two stable lightest-SUSY-particle (LSP) dark-matter candidates are produced, which escape without detection and lead to large missing transverse energy $E_{\mathrm{T}}^{\mathrm{miss}}$ in the event.

In this paper, we present several dedicated searches for chargino-neutralino pair production. The data, corresponding to an integrated luminosity of $4.98 \pm 0.11 \mathrm{fb}^{-1}$ [14] of proton-proton collisions at $\sqrt{s}=7 \mathrm{TeV}$, were collected by the Compact Muon Solenoid (CMS) experiment at the LHC in 2011. Even with the smaller cross sections of electroweak production, this data sample is sufficient to probe the production of charginos and neutralinos with masses well beyond existing constraints [15-22]. Since LHC studies have as yet found no evidence for new strongly interacting particles, we focus on scenarios in which such particles do not participate, and in which the final states are rich in leptons produced via intermediate states including sleptons (SUSY partners of the leptons, including sneutrinos, partners of neutrinos). These scenarios include cases such as those shown in figures 1 and 2, which are labeled using SUSY nomenclature, though the interpretation naturally extends to other BSM models. In the SUSY nomenclature, $\widetilde{\chi}_{1}^{0}$ is the lightest neutralino, presumed to be the LSP, and $\widetilde{\chi}_{2}^{0}$ and $\widetilde{\chi}_{3}^{0}$ are heavier neutralinos; $\widetilde{\chi}_{1}^{ \pm}$ is the lightest chargino. In figure 1 the slepton mass $m_{\tilde{\ell}}$ is less than the masses $m_{\widetilde{\chi}_{2}^{0}}$ and $m_{\widetilde{\chi}_{1}^{ \pm}}$, while in figure 2 it is greater, and the mass difference between the LSP and the next-lightest chargino or neutralino is large enough to lead to on-shell vector bosons. In addition to the dedicated searches, we leverage the results of some previous CMS SUSY searches [23-26], either by interpreting the previous results directly in the context of the scenarios in figures 1 and 2 , or by modifying the previous studies so that they target electroweak, rather than strong, production processes. Throughout this paper, "lepton" refers to a charged lepton; in specified contexts, it refers more specifically to an experimentally identified electron or muon.

To quantify our results, we present them in the context of simplified model spectra (SMS) [27-34]. SUSY models with bino-like $\widetilde{\chi}_{1}^{0}$ and wino-like $\widetilde{\chi}_{2}^{0}$ and $\widetilde{\chi}_{1}^{ \pm}$lead to the SMS trilepton signature of figure 1 , and motivate the simplifying assumption that the latter two gauginos have similar masses as a result of belonging to the same gauge group multiplet. We thus set $m_{\widetilde{\chi}_{2}^{0}}=m_{\widetilde{\chi}_{1}^{ \pm}}$, and present results as a function of this common mass and the LSP mass $m_{\widetilde{\chi}_{1}^{0}}$. The results for figure 1 depend also on the mass $m_{\tilde{\ell}}$ of the intermediate slepton (if left-handed, taken to be the same for its sneutrino $\widetilde{\nu}$ ), parametrized in terms of 


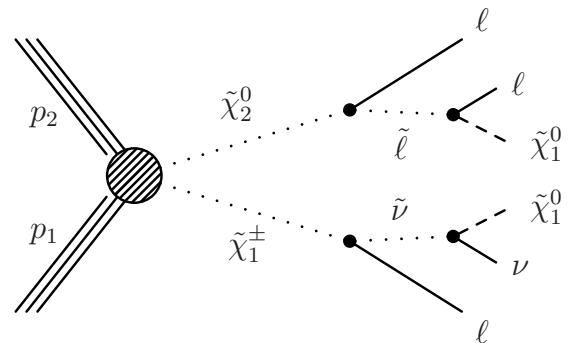

(a)

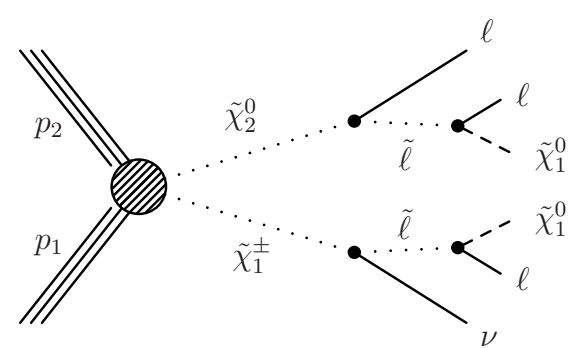

(b)

Figure 1. Diagrams of chargino-neutralino pair production in proton-proton collisions followed by decays leading to a final state with three leptons, two LSPs, and a neutrino. For left-handed sleptons (with accompanying sneutrinos), both diagrams exist, and for each diagram there is an additional diagram with $\widetilde{\chi}_{2}^{0} \rightarrow \ell \widetilde{\ell} \rightarrow \ell \ell \widetilde{\chi}_{1}^{0}$ replaced by $\widetilde{\chi}_{2}^{0} \rightarrow \widetilde{\nu} \nu \rightarrow \nu \nu \widetilde{\chi}_{1}^{0}$. Thus only $50 \%$ of produced pairs results in three leptons. For right-handed sleptons, only the right diagram exists, and $100 \%$ of produced pairs result in three leptons. In these diagrams and those of figure 2, dotted lines represent unstable intermediate states, and the dashed lines represent the LSP.

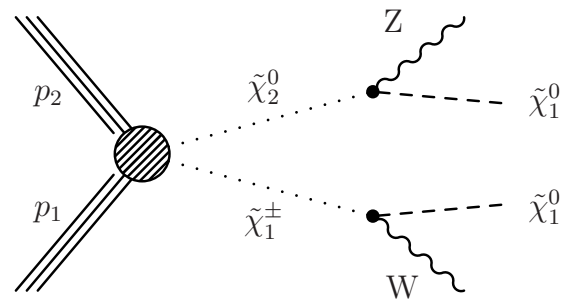

(a)

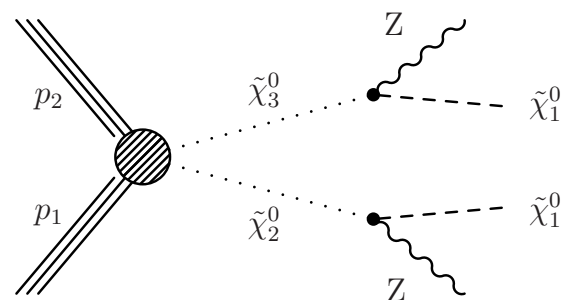

(b)

Figure 2. Diagrams of chargino-neutralino and neutralino-neutralino pair production in protonproton collisions followed by decay to on-shell $\mathrm{W}$ or Z bosons and LSPs.

a variable $x_{\tilde{\ell}}$ as

$$
m_{\widetilde{\ell}}=m_{\widetilde{\chi}_{1}^{0}}+x_{\widetilde{\ell}}\left(m_{\widetilde{\chi}_{1}^{ \pm}}-m_{\widetilde{\chi}_{1}^{0}}\right),
$$

where $0<x_{\tilde{\ell}}<1$. We present results for $x_{\tilde{\ell}}$ equal to 0.5 (i.e., the slepton mass equal to the mean of the LSP and chargino masses). In some cases we also present results for $x_{\widetilde{\ell}}=0.25$ and 0.75 .

The interpretation of the result may further depend on whether the sleptons are the SUSY partner $\tilde{\ell}_{L}$ or $\tilde{\ell}_{R}$ of left-handed or right-handed leptons. We consider two limiting cases. In one case, $\widetilde{\ell}_{R}$ does not participate while $\widetilde{\ell}_{L}$ and $\widetilde{\nu}$ do: then both diagrams of figure 1 exist, and the chargino and neutralino decay to all three lepton flavors with equal probability. Furthermore, two additional diagrams with $\widetilde{\chi}_{2}^{0} \rightarrow \ell \widetilde{\ell} \rightarrow \ell \ell \widetilde{\chi}_{1}^{0}$ replaced by $\widetilde{\chi}_{2}^{0} \rightarrow \widetilde{\nu} \nu \rightarrow \nu \nu \widetilde{\chi}_{1}^{0}$ reduce the fraction of three-lepton final states by $50 \%$. In the second case, in which $\widetilde{\ell}_{R}$ participates while $\widetilde{\ell}_{L}$ and $\widetilde{\nu}$ do not, only the diagram of figure 1(b) exists, and there is no $50 \%$ loss of three-lepton final states. Because the $\widetilde{\ell}_{R}$ couples to the chargino via its higgsino component, chargino decays to $\tilde{\ell}_{R}$ strongly favor the $\tau$ as the lepton. For the leptonic decay products, we thus consider primarily two flavor scenarios:

- The "flavor-democratic" scenario: the chargino $\left(\widetilde{\chi}_{1}^{ \pm}\right)$and neutralino $\left(\widetilde{\chi}_{2}^{0}\right)$ both decay with equal probability into all three lepton flavors, as expected for $\tilde{\ell}_{L}$; 
- The " $\tau$-enriched" scenario: the chargino decays exclusively to $\tau$ leptons as expected for $\widetilde{\ell}_{R}$, while the neutralino decays democratically.

With the selection criteria used in this paper, we have only limited sensitivity to a third scenario: the " $\tau$-dominated" scenario in which the chargino and neutralino both decay only to a $\tau$ lepton.

We place limits on the pair production cross section times branching fraction in the above scenarios. In additional interpretations given below in terms of bounds on masses within SMS, the $50 \%$ branching fraction to three leptons is taken into account when appropriate in $\widetilde{\ell}_{L}$ cases. For $x_{\widetilde{\ell}}=0.5$, the kinematic conditions for the processes of figure 1 are identical for $\widetilde{\ell}_{L}$ and $\widetilde{\ell}_{R}$, and the respective limits are trivially related. For other values of $x_{\widetilde{\ell}}(0.25$ and 0.75$)$, differences in experimental acceptance may alter the relationship.

For results based on the diagrams of figure 2, we assume that sleptons are too massive to participate, so that the branching fractions to vector bosons are $100 \%$. Even with such an assumption, there is little sensitivity to the $\mathrm{ZZ}$ channel of figure 2 (b) in the context of models such as the minimal supersymmetric extension of the standard model (MSSM), where neutralino pair production is suppressed relative to neutralino-chargino production. Rather, for the ZZ signature, we consider a specific gauge-mediated supersymmetry breaking (GMSB) Z-enriched higgsino model [35-37] that enhances the $\mathrm{ZZ}+E_{\mathrm{T}}^{\text {miss }}$ final state.

Following a description of the data collection and reconstruction procedures in section 2, section 3 describes searches specifically aimed at the three-lepton final state of figure 1. Kinematic observables that can distinguish signal from background include [3841] $E_{\mathrm{T}}^{\text {miss }}$, the invariant mass $M_{\ell \ell}$ of the opposite-sign leptons, and the transverse mass $M_{\mathrm{T}}$ formed from one lepton and the $E_{\mathrm{T}}^{\text {miss }}$. A three-lepton search using $E_{\mathrm{T}}^{\text {miss }}$ is presented in section 3.1, while a complementary approach using $M_{\ell \ell}$ and $M_{\mathrm{T}}$ is presented in section 3.2. In these three-lepton searches, the leptons selected are electrons and muons. Sensitivity to $\tau$ leptons arises only through their leptonic decays.

The three-lepton searches lose sensitivity when the probability to detect the third lepton becomes low. In section 4, we describe a search based on exactly two reconstructed leptons with the same electric charge (same sign), which extends the sensitivity to the processes of figure 1. This study, a modification of the CMS search for SUSY described in ref. [26], includes hadronically decaying $\tau$ leptons in addition to electrons and muons. Section 5 describes a search for the on-shell $\mathrm{W}$ and $\mathrm{Z}$ boson production processes of figure 2 . This study is a modification of the CMS search for SUSY in the Z boson plus jets and $E_{\mathrm{T}}^{\mathrm{miss}}$ channel [25].

Section 6 presents an interpretation of these searches, in some cases combining several together, and including the four-lepton results of ref. [24]. Results of related searches have also been recently reported by the ATLAS collaboration [42, 43].

Finally, appendix A provides a parametrized function for the detection efficiency of physics objects used in the analysis in section 3.2. This function will enable estimation of sensitivities for BSM models not considered in this paper that yield three leptons in the final state. 


\section{Detector, online selection, and object selection}

The online event selections (trigger) and further offline object selections closely follow those described in ref. [24], and are briefly summarized here. Exceptions are noted below in the sections specific to each analysis.

The central feature of the CMS apparatus is a superconducting solenoid, of $6 \mathrm{~m}$ internal diameter, providing a magnetic field of $3.8 \mathrm{~T}$. Within the field volume are a silicon pixel and strip tracker, a crystal electromagnetic calorimeter (ECAL), and a brass/scintillator hadron calorimeter. Muons are measured in gas-ionization detectors embedded in the steel return yoke. Extensive forward calorimetry complements the coverage provided by the barrel and endcap detectors. A more detailed description can be found in ref. [44].

CMS uses a right-handed coordinate system, with the origin at the nominal interaction point, the $x$ axis pointing to the center of the LHC, the $y$ axis pointing upwards (perpendicular to the plane of the LHC ring), and the $z$ axis along the counterclockwise-beam direction. The polar angle $\theta$ is measured from the positive $z$ axis, and the azimuthal angle $\phi$ (in radians) is measured in the $x-y$ plane. The pseudorapidity $\eta$ is a transformation of the polar angle defined by $\eta=-\ln [\tan (\theta / 2)]$.

Events from pp interactions must satisfy the requirements of a two-level trigger system. The first level performs a fast selection for physics objects (jets, muons, electrons, and photons) above certain thresholds. The second level performs a full event reconstruction. Events in this analysis are primarily selected using double-lepton triggers that require at least one electron or muon with transverse momentum $p_{\mathrm{T}}>17 \mathrm{GeV}$, and another with $p_{\mathrm{T}}>8 \mathrm{GeV}$, with $|\eta|<2.5$ for electrons and $|\eta|<2.4$ for muons. For channels involving $\tau$ leptons, triggers are used that rely on significant hadronic activity and $E_{\mathrm{T}}^{\mathrm{miss}}$, in addition to the presence of a single lepton or two hadronic $\tau$ candidates [26]. Additional triggers are used for calibration and efficiency studies.

Simulated event samples are used to study the characteristics of signal and standard model (SM) background. Most of the simulated event samples are produced with the MADGRAPH 5.1.1 [45, 46] event generator, with parton showering and hadronization performed with the PYTHIA 8.1 [47] program. Signal samples are generated with PYTHIA 6.424 [47]. The samples are generated using the CTEQ 6L1 [48] parton distribution functions. For the diboson backgrounds, MCFM [49] samples are used to help assess the theoretical uncertainties on the simulated samples. For the simulated SM samples, we use the most accurate calculations of the cross sections available, generally with next-to-leading order (NLO) accuracy $[50,51]$. The files specifying the SUSY signal model parameters are generated according to the SUSY Les Houches accord [52] standards with the ISAJET program [53], with cross sections calculated in PYTHIA to leading order and NLO corrections calculated using Prospino 2.1 [54]. Depending on the simulated sample, the detector response and reconstruction are modeled either with the CMS fast simulation framework [55], or with the GEANT4 [56] program, followed by the same event reconstruction as that used for data.

Events are reconstructed offline using the particle-flow (PF) algorithm [57, 58], which provides a self-consistent global assignment of momenta and energies. Details of the reconstruction and identification are given in refs. $[59,60]$ for electrons and muons. Leptonically 
decaying $\tau$ leptons are included in the selection of electrons or muons. In the same-sign dilepton search, hadronic $\tau$ lepton decays are identified with the "hadrons-plus-strips" algorithm [26, 61]. This algorithm combines PF photons and electrons into strips (caused by azimuthal bending of an electromagnetic shower in the CMS magnetic field) in order to reconstruct neutral pions. The neutral pions are combined with charged hadrons to reconstruct exclusive hadronic $\tau$ decay topologies. In the four-lepton results from ref. [24] used in the interpretations in section 6 , hadronic $\tau$ candidates are identified as isolated tracks with associated ECAL energy deposits consistent with those from neutral pions.

We consider events that contain electrons, muons, and (for a subset of the searches, as specified above) hadronically decaying $\tau$ leptons, each associated with the same primary vertex. Offline requirements on the lepton $p_{\mathrm{T}}$ and $\eta$ are described in the analysisspecific sections below. To reduce contamination due to leptons from heavy-flavor decays or misidentified hadrons in jets, an isolation criterion is formed by summing the track $p_{\mathrm{T}}$ and calorimeter $E_{\mathrm{T}}$ values in a cone of $\Delta R=0.3\left(0.4\right.$ for electrons in the three-lepton $+E_{\mathrm{T}}^{\text {miss }}$ search) around the lepton, where $\Delta R=\sqrt{(\Delta \phi)^{2}+(\Delta \eta)^{2}}$. The candidate lepton is excluded from the isolation sum. This sum is divided by the lepton's $p_{\mathrm{T}}$ to obtain the isolation ratio $I_{\text {rel }}$, which is required to be less than 0.15 .

Jets are reconstructed with the anti- $k_{\mathrm{T}}$ clustering algorithm [62] with a distance parameter of 0.5 . The jet reconstruction is based on PF objects. With exceptions noted below, jets are required to have $|\eta|<2.5$ and $p_{\mathrm{T}}>40 \mathrm{GeV}$ and to be separated from any lepton satisfying the analysis selection by $\Delta R>0.3$. Where applicable to suppress background from heavy flavors, we identify jets with b quarks (referred to throughout as "b jets") by using the CMS "track-counting high-efficiency algorithm" (TCHE) [63], which provides a b-jet tagging efficiency of $76 \%(63 \%)$ with a misidentification rate of $13 \%(2 \%)$ for the loose (medium) working point.

Events with an opposite-sign same-flavor (OSSF) dilepton (i.e., dielectron or dimuon) with invariant mass below $12 \mathrm{GeV}$ are rejected, to exclude quarkonia resonances, low-mass continuum, and photon conversions.

\section{Searches in the three-lepton final state}

For the searches in the three-lepton final state, we use reconstructed leptons identified as electrons and muons; any sensitivity to $\tau$ leptons comes indirectly through their leptonic decays. The main SM backgrounds in the three-lepton final state are from WZ production with three genuine isolated leptons that are "prompt" (created at the primary vertex), and from $t \bar{t}$ production with two such leptons and a third particle identified as such but that is "non-prompt" (created at a secondary vertex, as from a heavy-flavor decay) or not a lepton. We consider two complementary variants of this search. The first uses the missing transverse energy $E_{\mathrm{T}}^{\text {miss }}$ directly, and has slightly better sensitivity than the second when the difference between $m_{\widetilde{\chi}_{2}^{0}}=m_{\widetilde{\chi}_{1}^{ \pm}}$and the LSP mass $m_{\widetilde{\chi}_{1}^{0}}$ is large. The second search uses $E_{\mathrm{T}}^{\text {miss }}$ indirectly through the transverse mass $M_{\mathrm{T}}$, which is particularly effective in discriminating background from leptonic decays of $\mathrm{W}$ bosons in events with lower $E_{\mathrm{T}}^{\text {miss}}$; this search has more sensitivity than the first as $m_{\widetilde{\chi}_{1}^{0}}$ approaches $m_{\widetilde{\chi}_{2}^{0}}=m_{\widetilde{\chi}_{1}^{ \pm}}$. 


\subsection{Searches with three leptons using $E_{\mathrm{T}}^{\text {miss }}$ shape}

For our study of three-lepton events with significant $E_{\mathrm{T}}^{\text {miss }}$, we make use of our previous analysis [24], based on the same data sample as the present study. The analysis requires three leptons (only electrons or muons) and $H_{\mathrm{T}}<200 \mathrm{GeV}$, where $H_{\mathrm{T}}$ is the scalar sum of the $p_{\mathrm{T}}$ of the jets in the event. OSSF dileptons are rejected if $75 \mathrm{GeV}<M_{\ell \ell}<105 \mathrm{GeV}$ in order to suppress background from $\mathrm{Z}$ bosons. For the lepton selection, at least one electron or muon is required with $p_{\mathrm{T}}>20 \mathrm{GeV}$, and another with $p_{\mathrm{T}}>10 \mathrm{GeV}$; the third lepton must have $p_{\mathrm{T}}>8 \mathrm{GeV}$; this search additionally requires $|\eta|<2.1$ for all three leptons. A more detailed description of the analysis can be found in ref. [24].

The number of events observed for $E_{\mathrm{T}}^{\text {miss }}>50 \mathrm{GeV}$ and the corresponding background predictions are given in table 1 in $10-\mathrm{GeV}$-wide bins (corresponding to the display of the same data in figure 3 (left) of ref. [24]). The analysis in ref. [24] considers two regions of $E_{\mathrm{T}}^{\text {miss }}$ only: $E_{\mathrm{T}}^{\text {miss }}<50 \mathrm{GeV}$ and $E_{\mathrm{T}}^{\text {miss }}>50 \mathrm{GeV}$. In the present study, we take this latter region and use the separate contents of the bins in table 1 in a combined statistical treatment. This approach provides more powerful discrimination between signal and background than the treatment of ref. [24], because of the different shapes of signal and background across these bins.

All details of the event selection, background estimates, and evaluation of systematic uncertainties are as described in section 2 and ref. [24]. Briefly, efficiencies of electron/muon identification and isolation requirements are estimated using the method described in ref. [64] for $\mathrm{Z} \rightarrow \ell^{+} \ell^{-}$events, and are in agreement with the simulation to within $2 \%$ (1\%) for electrons (muons). Background due to Drell-Yan processes (including $\mathrm{Z}+$ jets boson production), with a jet providing a third genuine (non-prompt) lepton or a hadron misidentified as a lepton, is evaluated from studies of isolated tracks failing or passing electron/muon identification criteria, separately for samples enriched in heavy- and light-flavor jets. This background decreases rapidly to negligible levels for $E_{\mathrm{T}}^{\mathrm{miss}}>50 \mathrm{GeV}$. The main backgrounds for $E_{\mathrm{T}}^{\text {miss }}>50 \mathrm{GeV}$ are from diboson and $\mathrm{t} \overline{\mathrm{t}}$ production and are estimated from the simulation.

Section 6 presents the detailed interpretation of these results.

\subsection{Searches with three leptons using $M_{\ell \ell}$ and $M_{\mathrm{T}}$}

The alternative three-lepton search, based on $M_{\ell \ell}$ and $M_{\mathrm{T}}$, introduces in addition a veto on events having an identified b jet (using the TCHE medium working point) with $p_{\mathrm{T}}>$ $20 \mathrm{GeV}$. By vetoing only b jets, this requirement suppresses t $\overline{\mathrm{t}}$ background while avoiding exposure to signal loss (for example due to initial-state radiation) from a more general jet veto.

We require at least one electron or muon with $p_{\mathrm{T}}>20 \mathrm{GeV}$ and two more with $p_{\mathrm{T}}>$ $10 \mathrm{GeV}$, all with $|\eta|<2.4$. After requiring $E_{\mathrm{T}}^{\text {miss }}>50 \mathrm{GeV}$ (and making no requirement on $H_{\mathrm{T}}$ ), events are characterized by the values of the invariant mass $M_{\ell \ell}$ of the OSSF pair, and the transverse mass $M_{\mathrm{T}}$ formed from the $E_{\mathrm{T}}^{\text {miss }}$ vector and the transverse momentum $p_{\mathrm{T}}^{\ell}$ of the remaining lepton:

$$
M_{\mathrm{T}} \equiv \sqrt{2 E_{\mathrm{T}}^{\mathrm{miss}} p_{\mathrm{T}}^{\ell}\left[1-\cos \left(\Delta \phi_{\ell, E_{\mathrm{T}}^{\mathrm{miss}}}\right)\right]} .
$$




\begin{tabular}{|lcc|}
\hline$E_{\mathrm{T}}^{\text {miss }}$ Range $(\mathrm{GeV})$ & Observation & Background \\
\hline $50-60$ & 5 & $7.01 \pm 2.15$ \\
$60-70$ & 10 & $5.36 \pm 1.46$ \\
$70-80$ & 2 & $3.35 \pm 0.93$ \\
$80-90$ & 5 & $2.52 \pm 0.68$ \\
$90-100$ & 1 & $2.14 \pm 0.56$ \\
$100-110$ & 0 & $2.37 \pm 0.83$ \\
$110-120$ & 3 & $1.49 \pm 0.47$ \\
$120-130$ & 1 & $1.06 \pm 0.32$ \\
$130-140$ & 0 & $0.38 \pm 0.11$ \\
$140-150$ & 2 & $0.26 \pm 0.10$ \\
$150-160$ & 0 & $0.15 \pm 0.06$ \\
$160-170$ & 1 & $0.16 \pm 0.06$ \\
$170-180$ & 0 & $0.08 \pm 0.03$ \\
$180-190$ & 0 & $0.54 \pm 0.42$ \\
$190-200$ & 0 & $0.05 \pm 0.03$ \\
$>200$ & 0 & $0.33 \pm 0.16$ \\
\hline
\end{tabular}

Table 1. The observed and mean expected background in bins of $E_{\mathrm{T}}^{\mathrm{miss}}$ for three-lepton events with $H_{\mathrm{T}}<200 \mathrm{GeV}$, an opposite-sign same-flavor (OSSF) lepton pair, and no Z boson candidate. These results correspond to the distributions shown in figure 3 (left) of ref. [24]. Uncertainties include statistical and systematic contributions.

For three-muon and three-electron events, the OSSF pair with $M_{\ell \ell}$ closer to the $\mathrm{Z}$ mass is used. For backgrounds where a true OSSF pair arises from a low-mass virtual photon, this can result in a misassignment; simulation of this effect is validated with identified $\mu \mu \mathrm{e}$ and $\mu$ ee events by treating all three leptons as having the same flavor.

\subsubsection{Background due to $\mathrm{WZ}$ production}

The largest background is due to SM WZ production in which both bosons decay leptonically. Studies with data indicate that the simulation-based estimates of systematic uncertainties on both the WZ background characteristics and signal resolutions are generally reliable, but especially at high- $M_{\mathrm{T}}$, corrections are obtainable through detailed comparisons of data and the simulation. Here, we present one such study: the calibration of the hadronic recoil of the WZ system. In addition, the overall WZ event yield normalization is validated using events where $M_{\ell \ell}$ and $M_{\mathrm{T}}$ are consistent with the $\mathrm{Z}$ and $\mathrm{W}$ boson masses $\left(81 \mathrm{GeV}<M_{\ell \ell}<101 \mathrm{GeV}, M_{\mathrm{T}}<100 \mathrm{GeV}\right)$, respectively. We find good agreement with the SM simulations, as presented below.

The simulation of $E_{\mathrm{T}}^{\text {miss }}$ (and hence $M_{\mathrm{T}}$ ) is corrected using a generalization of the Z-recoil method used in the CMS measurements of the $\mathrm{W}$ and $\mathrm{Z}$ cross sections [64]. The transverse hadronic recoil vector $\vec{u}_{\mathrm{T}}$ is

$$
\vec{u}_{\mathrm{T}}=-\vec{E}_{\mathrm{T}}^{\mathrm{miss}}-\vec{p}_{\mathrm{T}, 1}-\vec{p}_{\mathrm{T}, 2}
$$




\begin{tabular}{|l|ccc|}
\hline & On-Z, high- $M_{\mathrm{T}}$ & Off-Z, low- $M_{\mathrm{T}}$ & Off-Z, high- $M_{\mathrm{T}}$ \\
\hline Hadronic recoil & $29.7 \%$ & $0.9 \%$ & $14.9 \%$ \\
WZ versus Z recoil & $7.2 \%$ & $0.5 \%$ & $3.4 \%$ \\
Lepton energy scale & $1.8 \%$ & $0.7 \%$ & $0.7 \%$ \\
Lepton energy resolution & $1.4 \%$ & $6.9 \%$ & $4.5 \%$ \\
Boson $p_{\mathrm{T}}$ & $5.1 \%$ & $0.4 \%$ & $2.2 \%$ \\
Z mass shape & $0.2 \%$ & $0.4 \%$ & $2.5 \%$ \\
Normalization & $9.3 \%$ & $9.3 \%$ & $9.3 \%$ \\
\hline Sum & $32.4 \%$ & $11.7 \%$ & $18.8 \%$ \\
\hline
\end{tabular}

Table 2. Relative systematic uncertainties for the mean WZ background. "On-Z" refers to events in which the OSSF pair satisfies $81<M_{\ell \ell}<101 \mathrm{GeV}$. "Off-Z" refers to events with either $M_{\ell \ell}<$ $81 \mathrm{GeV}$ or $M_{\ell \ell}>101 \mathrm{GeV}$. The events are further categorized according to whether they have low $(<100 \mathrm{GeV})$ or high $(>100 \mathrm{GeV}) M_{\mathrm{T}}$ values. The "Off- $Z$, low- $M_{\mathrm{T}}$ " column corresponds to the sum of events in regions I and V in figure 3, while the "Off-Z, high $M_{\mathrm{T}}$ " column corresponds to the sum of regions II and IV.

for $\mathrm{Z}$ events and

$$
\vec{u}_{\mathrm{T}}=-\vec{E}_{\mathrm{T}}^{\mathrm{miss}}-\vec{p}_{\mathrm{T}, 1}-\vec{p}_{\mathrm{T}, 2}-\vec{p}_{\mathrm{T}, 3}
$$

for WZ events, where $\vec{E}_{\mathrm{T}}^{\text {miss }}$ is the missing transverse energy vector, and $\vec{p}_{\mathrm{T}, i}$ is the transverse momentum vector of each of the two leptons from the $\mathrm{Z}$ decay or three leptons from the WZ decay. The recoil vector is resolved into components: $u_{1}$ parallel to the direction of the respective $\mathrm{Z}$ or $\mathrm{WZ}$ system, and $u_{2}$ perpendicular to the $\mathrm{Z}$ or $\mathrm{WZ}$ direction (known in the simulation and approximated in the data). The $u_{1}$ component is sensitive to calorimeter response and resolution, while the $u_{2}$ component is predominantly determined by the underlying event and multiple interactions. Using a pure sample of $\mathrm{Z}$ boson events, detailed studies of both components as a function of the $\mathrm{Z}$ boson $p_{\mathrm{T}}$ value yield corrections to the simulation, which are implemented event-by-event assuming that the results for $\mathrm{Z}$ production are similar to those for WZ production. These data-based corrections alter the expected background by up to $25 \%$, and allow us to reduce the systematic uncertainty associated with the simulation.

Reconstructed leptonic decays of $\mathrm{Z}$ bosons are used to calibrate lepton energy scales and resolutions, separately for electrons and muons, in bins of $p_{\mathrm{T}}$ and $\eta$. The uncertainties from this procedure are propagated into uncertainties on the mean background estimation by using the simulation. Table 2 summarizes these and the other systematic uncertainties in the estimation of the WZ background.

\subsubsection{Background due to $t \overline{\mathrm{t}}$ production and other processes}

The second-largest background is from events with two genuine isolated prompt leptons and a third identified lepton that is either a non-prompt genuine lepton from a heavyflavor decay or a misidentified hadron, typically from a light-flavor jet. Top-quark pair, $\mathrm{Z}+$ jets, and $\mathrm{WW}+$ jets events are the main processes that contribute to this background. We measure this background using control samples in data. The probability for a non- 
prompt lepton to satisfy the isolation requirement $\left(I_{\text {rel }}<0.15\right)$ is measured in a data sample enriched with QCD dijet events, and varies from $2 \%$ to $3 \%$ for muons and from $6 \%$ to $8 \%$ for electrons as a function of lepton $p_{\mathrm{T}}$. These probabilities, applied to the three-lepton events where the isolation requirement on one of the leptons is removed, are used to estimate background due to such non-prompt leptons.

Another background studied with data is the rare process in which a $\mathrm{Z}$ boson is accompanied by an initial- or final-state radiation photon that converts internally or externally, leading to a reconstructed three-lepton final state when the conversion is highly asymmetric [24].

The systematic uncertainties assigned to the tet background and other backgrounds studied with data are based on differences between the predicted and true yields when the method is applied to simulated events, as well as on the effect of the prompt-lepton contamination in control samples.

Backgrounds from very rare SM processes that have not yet been adequately measured

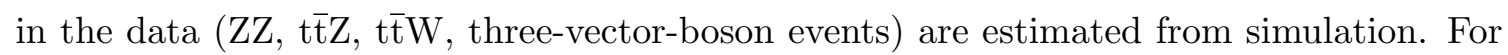
these sources, a systematic uncertainty of $50 \%$ is assigned to account for uncertainty in the NLO calculations of cross sections.

\subsubsection{Observations in the three-lepton search with $M_{\ell \ell}$ and $M_{\mathrm{T}}$}

Figure 3 presents a scatter plot of $M_{\mathrm{T}}$ versus $M_{\ell \ell}$ for the selected events. The dashed lines divide the plane into six regions. The horizontal dashed line at $M_{\mathrm{T}}=100 \mathrm{GeV}$ separates the lower- $M_{\mathrm{T}}$ region, which contains most of the background associated with on-shell $\mathrm{W}$ bosons, from the region depleted of this background. The vertical dashed lines at $M_{\ell \ell}=81 \mathrm{GeV}$ and $101 \mathrm{GeV}$ define the endpoints of the region dominated by $\mathrm{Z}$ boson decays. In the lower $M_{\ell \ell}$ region, the search is sensitive to the signal production process of figure 1 with small to moderate $\widetilde{\chi}_{2}^{0}-\widetilde{\chi}_{1}^{0}$ mass splittings $(<100 \mathrm{GeV})$, while being subject to background from $W+\gamma^{*} / Z^{*}$ events, especially in Region I. In the higher- $M_{\ell \ell}$ region, the search is sensitive to models with larger mass splittings. Region VI (on-Z, low $M_{\mathrm{T}}$ ) is dominated by WZ and ZZ backgrounds. Leakage from this region contaminates the nearby regions.

Figure 4 shows the $M_{\mathrm{T}}$ distributions for data and the mean expected SM background below the Z (Regions I and II), on-Z (Regions III and VI), and above the Z (Regions IV and $\mathrm{V}$ ). The background shape from non-prompt or misidentified leptons is taken from simulation while the normalization is derived from the data.

Table 3 contains a summary of the mean estimated backgrounds and observed yields. There is no evidence for a signal, and the background shape is well reproduced within the limited statistics.

Section 6 contains the detailed interpretation of these observations, which are found to have comparable sensitivity to the $E_{\mathrm{T}}^{\text {miss }}$-based search of section 3.1.

\section{Searches in the same-sign two-lepton final state}

Three-lepton final states are not sensitive to direct chargino-neutralino production if one of the leptons is unidentified, not isolated, or outside the acceptance of the analysis. The 


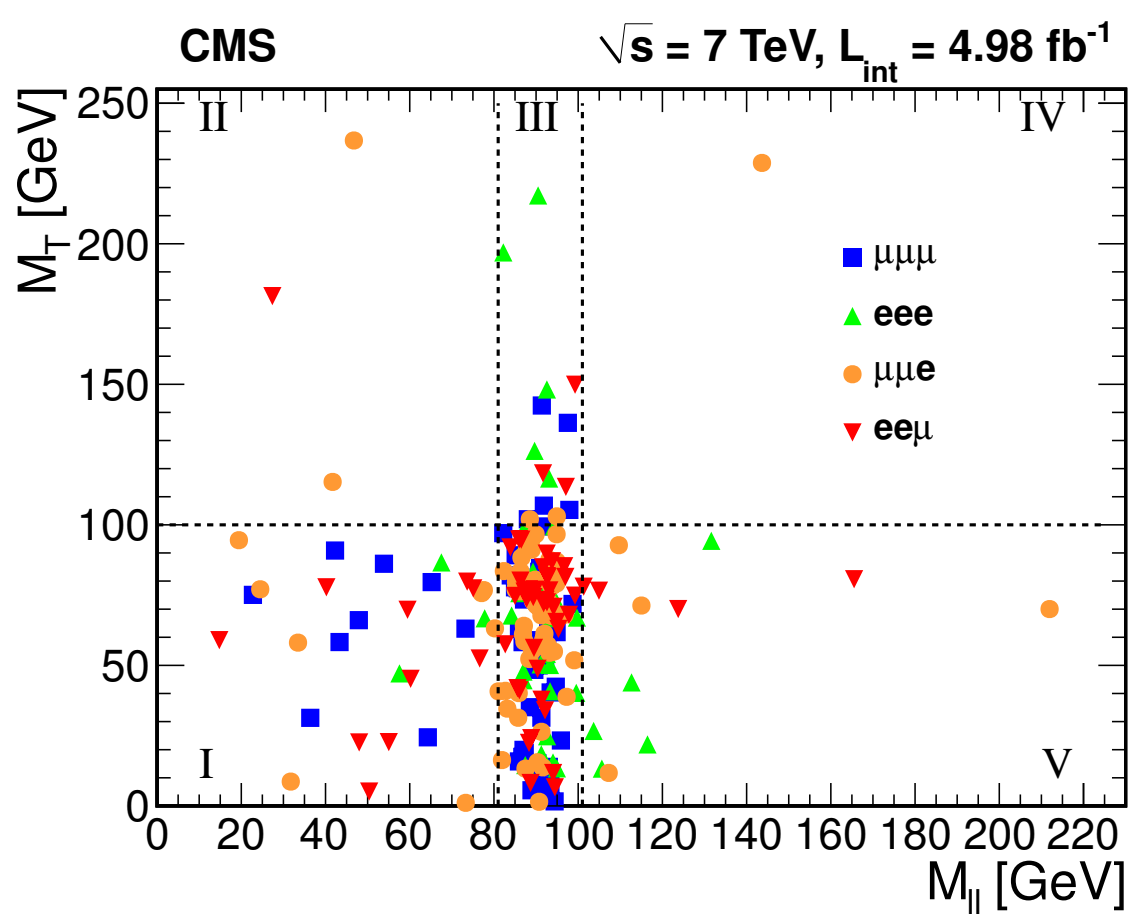

Figure 3. $M_{\mathrm{T}}$ versus $M_{\ell \ell}$ for the selected events in data. (Unlabeled Region VI lies between Regions I and V.) Two events appear outside the limits of the plot; one is a $\mu \mu \mu$ event at $\left(M_{\ell \ell}, M_{\mathrm{T}}\right)$ $=(240 \mathrm{GeV}, 399 \mathrm{GeV})$ and the other is an eee event at $(95 \mathrm{GeV}, 376 \mathrm{GeV})$.

\begin{tabular}{|cccccc|}
\hline Region & WZ & Non-prompt & Rare SM & Total background & Data \\
\hline I & $16.2 \pm 2.9$ & $4.7 \pm 2.4$ & $2.1 \pm 1.5$ & $23.0 \pm 5.1$ & 31 \\
II & $3.6 \pm 0.8$ & $1.94 \pm 1.02$ & $0.4 \pm 0.2$ & $6.0 \pm 1.3$ & 3 \\
III & $15.6 \pm 5.7$ & $0.2 \pm 0.1$ & $0.8 \pm 0.4$ & $16.6 \pm 5.7$ & 17 \\
IV & $1.6 \pm 0.4$ & $0.2 \pm 0.1$ & $0.4 \pm 0.2$ & $2.2 \pm 0.5$ & 2 \\
V & $8.7 \pm 1.7$ & $1.4 \pm 0.8$ & $0.9 \pm 0.4$ & $11.0 \pm 1.9$ & 12 \\
VI & $150.6 \pm 25.7$ & $2.6 \pm 1.4$ & $11.7 \pm 5.8$ & $164.9 \pm 26.4$ & 173 \\
\hline
\end{tabular}

Table 3. Summary of mean expected backgrounds and observations in each region for the three-lepton search based on $M_{\ell \ell}$ and $E_{\mathrm{T}}^{\text {miss }}$. Uncertainties include statistical and systematic contributions.

CMS detector has high geometrical acceptance for all leptons. However, when the signalmodel mass splittings are such that one lepton has $p_{\mathrm{T}}<10 \mathrm{GeV}$, three leptons are unlikely to be selected. Some of these otherwise-rejected events can be recovered by requiring only two leptons, which should however be of same sign (SS) to suppress the overwhelming background from opposite-sign dileptons [38, 65].

The SS dilepton search requires at least one electron or muon with $p_{\mathrm{T}}>20 \mathrm{GeV}$, and another with $p_{\mathrm{T}}>10 \mathrm{GeV}$, with $|\eta|<2.4$ for both. We exclude events that contain a third lepton, using the criteria of section 3.2, in order to facilitate combination with those results. Furthermore, as events with $\tau$ leptons can be important in some SUSY scenarios [66], we 


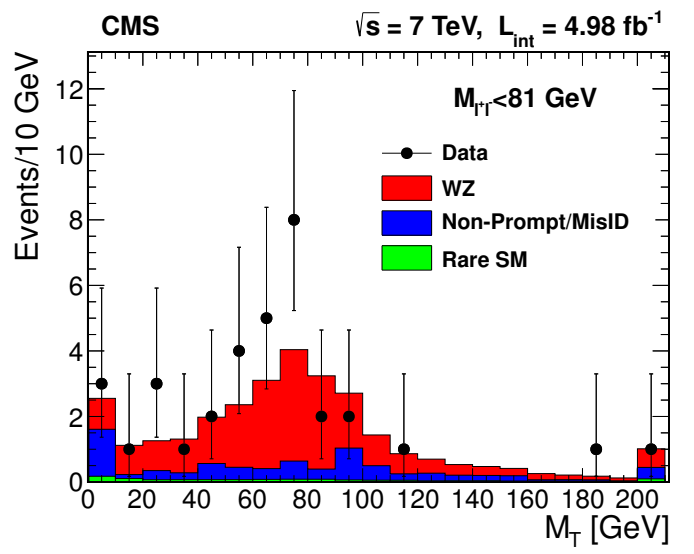

(a)

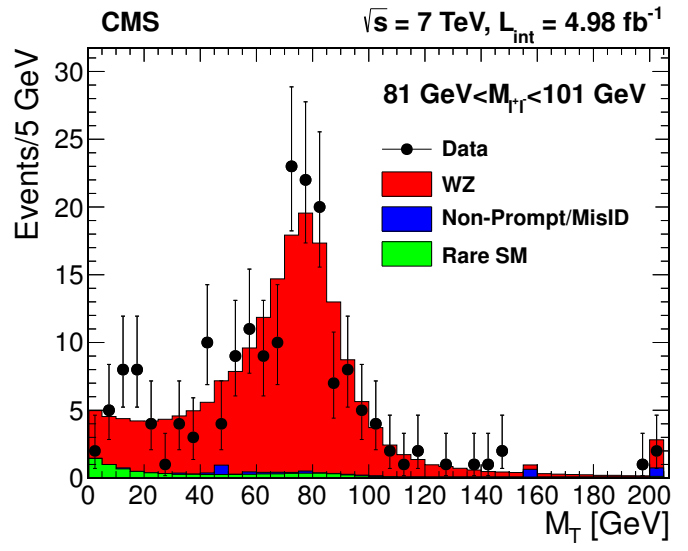

(b)

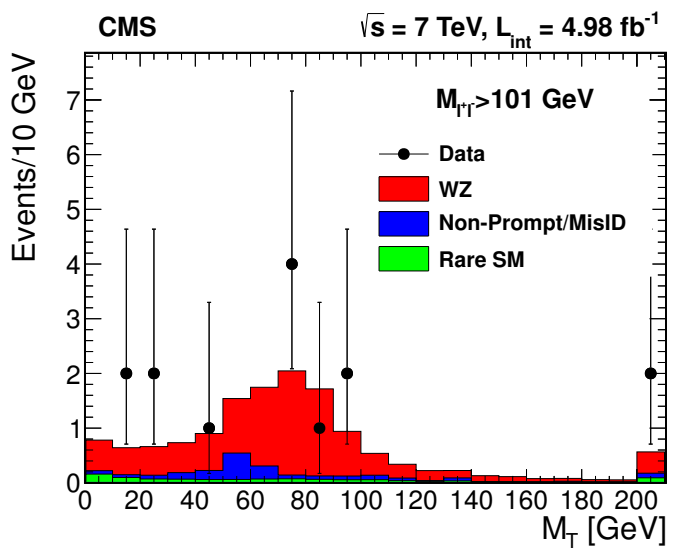

(c)

Figure 4. Observed and mean expected $M_{\mathrm{T}}$ distribution for $M_{\ell \ell}$ in the regions (a) below the Z boson mass, (b) on the $\mathrm{Z}$ boson mass, and (c) above the $\mathrm{Z}$ boson mass. Rare SM processes include three-vector-boson production, production of top-quark pairs together with a vector boson, and ZZ production. The last bin in each histogram includes the events with $M_{\mathrm{T}}$ beyond the histogram range.

include the e $\tau, \mu \tau$, and $\tau \tau$ final states; for this purpose, we use hadronic decays of the $\tau$. The isolation criteria for hadronically decaying $\tau$ leptons require that, apart from the hadronic decay products, there be no charged hadron or photon with $p_{\mathrm{T}}$ above $0.8 \mathrm{GeV}$ within a cone of $\Delta R=0.5$ around the direction of the $\tau$.

An important class of background for SS events is that with one genuine prompt lepton and either a non-prompt genuine lepton from a heavy-flavor decay or a misidentified hadron. This background arises mainly from events with jets and a $\mathrm{W}$ or $\mathrm{Z}$ boson. Much of the analysis strategy is driven by the need to suppress these events. Electron and muon selection criteria are thus tightened: the isolation criterion becomes $I_{\text {rel }}<0.1$, and we add a criterion to limit the maximum energy deposit of muon candidates in the calorimeters.

Events containing OSSF pairs with $\left|M_{\ell \ell}-M_{Z}\right|<15 \mathrm{GeV}$ are eliminated in order to reduce background due to processes such as $\mathrm{WZ}$ and $\mathrm{t} \overline{\mathrm{t} Z}$ production. For this purpose we select these events by using looser isolation criteria $\left(I_{\text {rel }}<1.0\right.$ for muons and bar- 


\begin{tabular}{|lccccccc|}
\hline Source & $\mathrm{ee}$ & $\mu \mu$ & $\mathrm{e} \mu$ & $\mathrm{e} \tau$ & $\mu \tau$ & $\tau \tau$ & Sum \\
\hline Non-pr/misID & $1.0 \pm 0.8$ & $0.0 \pm 0.2$ & $1.7 \pm 1.0$ & $1.5 \pm 1.1$ & $1.7 \pm 0.6$ & $0.00 \pm 0.00$ & $5.8 \pm 1.9$ \\
Charge misass & $0.0 \pm 0.0$ & - & $0.0 \pm 0.0$ & $0.0 \pm 0.0$ & $0.0 \pm 0.1$ & $0.00 \pm 0.01$ & $0.1 \pm 0.1$ \\
Rare SM & $1.0 \pm 0.7$ & $0.7 \pm 0.5$ & $1.3 \pm 0.7$ & $0.3 \pm 0.1$ & $0.4 \pm 0.2$ & $0.00 \pm 0.00$ & $3.7 \pm 1.5$ \\
\hline Total background & $2.1 \pm 1.0$ & $0.7 \pm 0.5$ & $3.1 \pm 1.2$ & $1.7 \pm 1.1$ & $2.0 \pm 0.6$ & $0.00 \pm 0.01$ & $9.5 \pm 2.4$ \\
\hline Observed & 2 & 1 & 0 & 1 & 1 & 0 & 5 \\
\hline
\end{tabular}

Table 4. Summary of mean expected backgrounds and observed yields in the $E_{\mathrm{T}}^{\text {miss }}>200 \mathrm{GeV}$ signal region for all six same-sign dilepton channels. The background categories comprise nonprompt and misidentified leptons, charge misassignment, and rare SM processes. Uncertainties include statistical and systematic contributions.

rel electrons, and $I_{\text {rel }}<0.6$ for endcap electrons) and looser identification requirements for electrons.

The remaining background with a non-prompt lepton is estimated with techniques described in ref. [26], where the probability for a non-prompt lepton to pass the signal selection is derived from control regions in data using extrapolations in the isolation and identification criteria. The systematic uncertainty on these predictions is $50 \%$ for light leptons and $30 \%$ for $\tau$ leptons.

Residual background is mostly due to charge misassignment in events with an OSSF pair, e.g., from Drell-Yan, t $\bar{t}$, or WW processes. We quantify the charge misassignment probability for electrons and $\tau$ leptons by studying SS ee or $\tau \tau$ events inside the $\mathrm{Z}$ mass peak region in data. For electrons, this probability is $0.0002 \pm 0.0001$ in the ECAL barrel and $0.0028 \pm 0.0004$ in the ECAL endcap, and for $\tau$ leptons it is $0.009 \pm 0.024$. For muons, it is determined from cosmic-ray data to be of order $10^{-5}$ and is neglected.

Backgrounds of lesser importance include those from rare SM processes such as diboson production, associated production of a t $\bar{t}$ pair with a vector boson, or triboson production. They are taken from simulation with a $50 \%$ systematic uncertainty assigned. An exception is WZ production, for which normalization to the measured cross section is available, thus reducing the systematic uncertainty to $20 \%$.

The distribution of events thus selected is studied in the plane of $E_{\mathrm{T}}^{\text {miss }}$ versus $H_{\mathrm{T}}$, as displayed in figure 5 (a). The signal region is defined by the criterion $E_{\mathrm{T}}^{\text {miss }}>200 \mathrm{GeV}$, with the $120 \mathrm{GeV}<E_{\mathrm{T}}^{\text {miss }}<200 \mathrm{GeV}$ interval used as a control region to confirm understanding of backgrounds. In the control region, the total mean expected background for events without a $\tau$ (ee, $\mu \mu$, and e $\mu$ events) is $24.8 \pm 7.6$, and 27 events are observed. The total mean expected background for $\mathrm{e} \tau, \mu \tau$, and $\tau \tau$ events is $24.5 \pm 8.9$, and 26 events are observed. The observed signal region yields in the various lepton-flavor final states are displayed in figure 5(b). Table 4 presents the mean expected background and the observed yields in the signal region. Section 6 presents the detailed interpretation of these observations; combining the same-sign dilepton search with the three-lepton search increases the mass limits by up to approximately $20 \mathrm{GeV}$.

The same-sign analysis is potentially sensitive to the processes of figure 1 in the $\tau$ dominated scenario, in which the chargino and neutralino both decay only to a $\tau$. With the present selection, we are only able to exclude a limited region of phase space for this scenario, bounded by $m_{\widetilde{\chi}_{1}^{0}}<50 \mathrm{GeV}$ and $m_{\widetilde{\chi}_{2}^{0}}=m_{\widetilde{\chi}_{1}^{ \pm}}<250 \mathrm{GeV}$. 


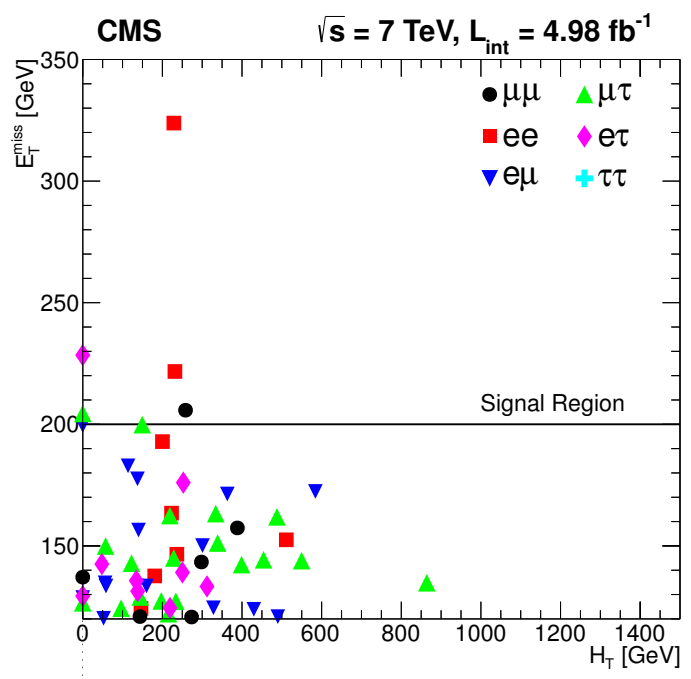

(a)

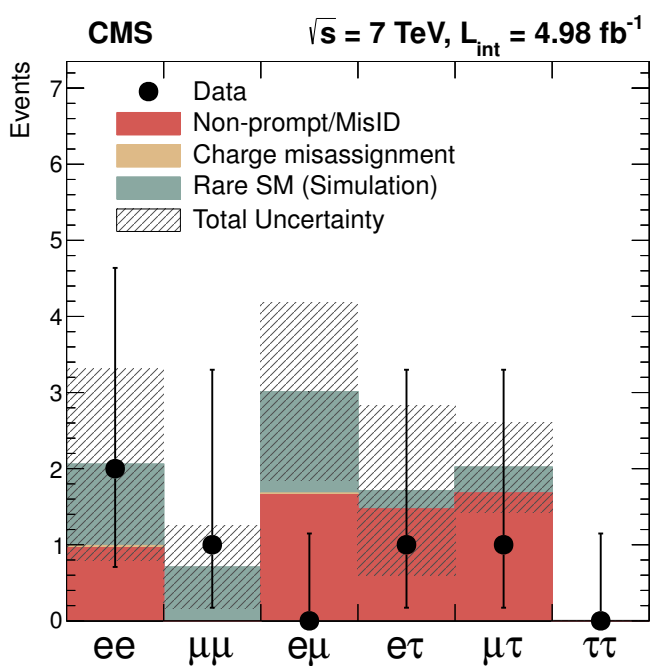

(b)

Figure 5. (a) $E_{\mathrm{T}}^{\text {miss }}$ versus $H_{\mathrm{T}}$ for same-sign dilepton candidate events. (b) Mean expected background yields with their uncertainty and observed number of events in the six channels, for the signal region $\left(E_{\mathrm{T}}^{\text {miss }}>200 \mathrm{GeV}\right)$.

\section{Searches in the $\mathrm{WZ} / \mathrm{ZZ}+E_{\mathrm{T}}^{\text {miss }}$ final state with two leptons and two jets}

Finally, we consider events with two on-shell vector bosons and significant $E_{\mathrm{T}}^{\text {miss }}$. Ref. [24] presents results relevant for the four-lepton final state, corresponding to the two-Z-boson process of figure $2(\mathrm{~b})$, when each $\mathrm{Z}$ boson decays either to an electron or a muon pair. In the following, we extend sensitivity to both diagrams of figure 2 by selecting events in which a $\mathrm{Z}$ boson decays to either ee or $\mu \mu$, while a $\mathrm{W}$ boson or another $\mathrm{Z}$ boson decays to two jets. SM diboson events with the corresponding final states do not contain intrinsic $E_{\mathrm{T}}^{\mathrm{miss}}$.

This search is an extension of our previous result [25]. We use the same selection of jets, leptons, and $E_{\mathrm{T}}^{\mathrm{miss}}$, as well as the same background estimation methods. Both leptons must have $p_{\mathrm{T}}>20 \mathrm{GeV}$. In particular, jets are required to have $p_{\mathrm{T}}>30 \mathrm{GeV}$ and $|\eta|<3$. The $E_{\mathrm{T}}^{\text {miss }}$ signal regions are indicated in table 5, with the entries indicating mean background estimates after applying all selection criteria described below.

We suppress background from t $\bar{t}$ events by a factor of approximately 10 by rejecting events that contain an identified $\mathrm{b}$ jet. We use the TCHE loose (medium) working point for jets with $p_{\mathrm{T}}<100 \mathrm{GeV}(>100 \mathrm{GeV})$. Further suppression of the t $\overline{\mathrm{t}}$ and $\mathrm{Z}+$ jets background is achieved by requiring that the dijet mass $M_{j j}$ be consistent with a $\mathrm{W}$ or $\mathrm{Z}$ boson, namely $70 \mathrm{GeV}<M_{j j}<110 \mathrm{GeV}$. Background from $\mathrm{WZ}+$ jets events is suppressed by rejecting events that contain a third identified lepton with $p_{\mathrm{T}}>20 \mathrm{GeV}$.

Background from $\mathrm{SM} \mathrm{Z}+$ jets events with artificial $E_{\mathrm{T}}^{\text {miss }}$ from jet mis-measurements must be carefully estimated, since the artificial $E_{\mathrm{T}}^{\text {miss }}$ is not necessarily well-reproduced in simulation. Using the method described in ref. [25], a control sample of $\gamma+$ jets events is 


\begin{tabular}{|lccc|}
\hline Source & $30 \leq E_{\mathrm{T}}^{\text {miss }}<60 \mathrm{GeV}$ & $60 \leq E_{\mathrm{T}}^{\text {miss }}<80 \mathrm{GeV}$ & $80 \leq E_{\mathrm{T}}^{\text {miss }}<100 \mathrm{GeV}$ \\
\hline$Z+$ jets background & $2298 \pm 737$ & $32.9 \pm 11.1$ & $5.2 \pm 1.8$ \\
OF background & $11 \pm 2$ & $6.6 \pm 1.6$ & $4.6 \pm 1.2$ \\
WZ/ZZ background & $50 \pm 25$ & $3.9 \pm 2.0$ & $2.2 \pm 1.1$ \\
\hline Total background & $2359 \pm 737$ & $43.4 \pm 11.4$ & $12.0 \pm 2.4$ \\
\hline Data & 2416 & 47 & 7 \\
\hline Source & $100 \leq E_{\mathrm{T}}^{\text {miss }}<150 \mathrm{GeV}$ & $150 \leq E_{\mathrm{T}}^{\text {miss }}<200 \mathrm{GeV}$ & $E_{\mathrm{T}}^{\text {miss }} \geq 200 \mathrm{GeV}$ \\
\hline Z + jets background & $1.7 \pm 0.6$ & $0.4 \pm 0.2$ & $0.20 \pm 0.09$ \\
OF background & $4.6 \pm 1.2$ & $0.8 \pm 0.3$ & $0.06 \pm 0.07$ \\
WZ/ZZ background & $2.5 \pm 1.3$ & $0.7 \pm 0.4$ & $0.4 \pm 0.2$ \\
\hline Total background & $8.8 \pm 1.8$ & $1.9 \pm 0.5$ & $0.7 \pm 0.3$ \\
\hline Data & 6 & 2 & 0 \\
\hline
\end{tabular}

Table 5. Summary of mean expected backgrounds and observed data in each of the $E_{\mathrm{T}}^{\text {miss }}$ signal regions, in final states with two opposite-sign leptons, two jets, and $E_{\mathrm{T}}^{\text {miss }}$. The total background is the sum of the $\mathrm{Z}+$ jets background evaluated with $\gamma+$ jets events, the flavor-symmetric background evaluated from opposite-flavor events (OF background), and the WZ/ZZ background expected from simulation (WZ/ZZ background). Uncertainties include statistical and systematic contributions.

used to model the $E_{\mathrm{T}}^{\text {miss }}$ in $\mathrm{Z}+$ jets events, after performing a reweighting procedure to take into account the different kinematic properties of the hadronic systems in the control and signal samples.

Background processes with uncorrelated flavor, while dominated by t $\overline{\mathrm{t}}$ events, also include events with $\tau \tau$ (via Drell-Yan production and followed by leptonic decays), WW, and single top production. For these processes, production in the same-flavor ee and $\mu \mu$ final states used for the search is modeled using a control sample of opposite-flavor (OF) $\mathrm{e} \mu$ events. Subdominant background contributions from SM WZ and ZZ production are estimated from simulation.

The mean expected backgrounds in bins of $E_{\mathrm{T}}^{\text {miss }}$ and the observed yields are summarized in table 5 and displayed in figure 6 . Section 6 contains the interpretation of these results, including a combination with those of ref. [24].

\section{$6 \quad$ Interpretations of the searches}

In this section, we present the interpretation of our results. Section 6.1 presents the limits on the SMS of figure 1 from the three-lepton search using the $E_{\mathrm{T}}^{\text {miss }}$ shape (section 3.1). Section 6.2 presents the limits on the same SMS from the three-lepton search using $M_{\ell \ell}$ and $M_{\mathrm{T}}$ (section 3.2), the same-sign dilepton search (section 4), and their combination. Section 6.3 presents the limits on the SMS of figure 2 using results from section 3 and from the $\mathrm{WZ}+E_{\mathrm{T}}^{\text {miss }}$ analysis of section 5 , as well as limits on a GMSB model using results from the $\mathrm{ZZ}+E_{\mathrm{T}}^{\mathrm{miss}}$ analysis of section 5 and the four-lepton results of ref. [24]. In all the search channels, the observations agree with the expected background.

We present upper limits on the cross sections for pair production of charginos and neutralinos. All upper limits are computed at $95 \%$ confidence level $(\mathrm{CL})$ using the $\mathrm{CL}_{\mathrm{s}}$ criterion [67, 68] with choices in the implementation following those in ref. [69]. Using 

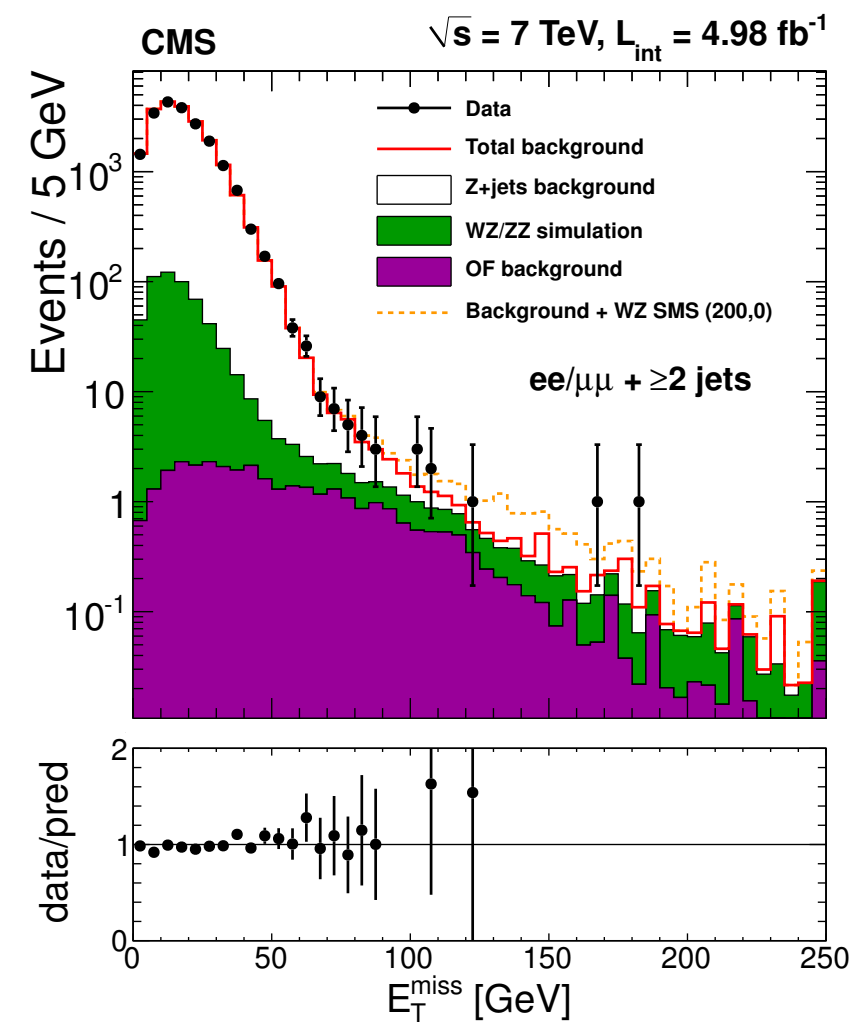

Figure 6. Observed $E_{\mathrm{T}}^{\text {miss }}$ distribution for $\mathrm{WZ}+E_{\mathrm{T}}^{\text {miss }}$ events after all selection criteria are applied except that on $E_{\mathrm{T}}^{\text {miss }}$ (solid points), in comparison with the corresponding SM expectation. For purposes of illustration, the $E_{\mathrm{T}}^{\text {miss }}$ distribution expected for WZ SMS events with $m_{\widetilde{\chi}_{2}^{0}}=m_{\tilde{\chi}_{1}^{ \pm}}=$ $200 \mathrm{GeV}$ and a massless LSP is shown. The plot below the main figure shows the ratio of the observed and mean-expected-background distributions.

the NLO cross section calculations from ref. [50, 51], we also evaluate 95\% CL exclusion curves. The exclusion curves are shown not only for their central values, but also when the NLO cross section is varied by \pm 1 standard deviation $(\sigma)$ of its uncertainty [51]. In addition, we display the median expected exclusion limit in an ensemble of experiments with background only, as well as the uncertainty band that contains $68 \%$ of the limits in the ensembles.

\subsection{Limits on SMS from the search with three leptons using $E_{\mathrm{T}}^{\text {miss }}$ shape}

Figure 7(a) displays the 95\% CL upper limits on the cross section times branching fraction in the $m_{\widetilde{\chi}_{1}^{0}}$ versus $m_{\widetilde{\chi}_{2}^{0}}\left(=m_{\widetilde{\chi}_{1}^{ \pm}}\right)$plane, with $x_{\widetilde{\ell}}=0.5$ in the flavor-democratic scenario described in the Introduction. The contour bounds the excluded region in the plane assuming the NLO cross section calculation and a $50 \%$ branching fraction to three leptons, as appropriate for this SMS. Figure 7(b) displays the corresponding limits for the $\tau$-enriched scenario. The lower-sensitivity feature in the curve, noticeable where the common mass $m_{\widetilde{\chi}_{2}^{0}}=m_{\widetilde{\chi}_{1}^{ \pm}}$is approximately $100 \mathrm{GeV}$ greater than $m_{\widetilde{\chi}_{1}^{0}}$, corresponds to the phase space where the dilepton mass has a high probability to be close to the $\mathrm{Z}$ mass, such that the event is rejected. 


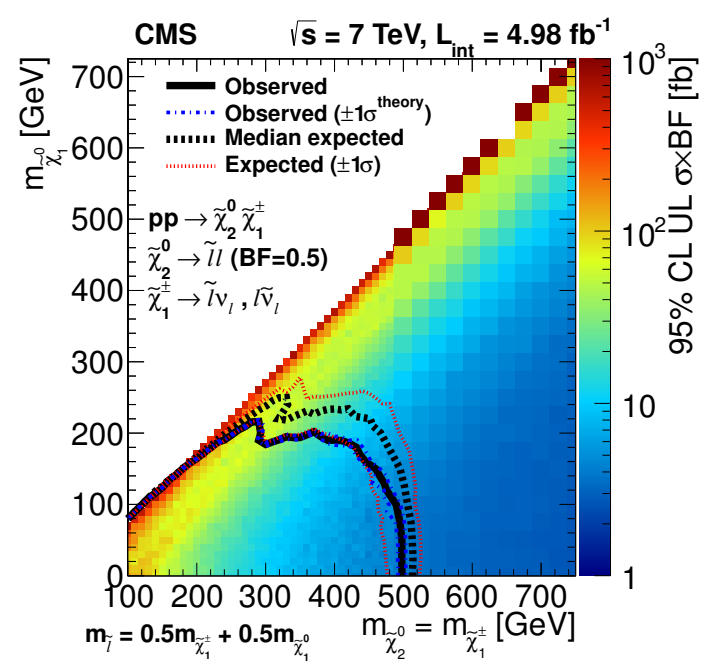

(a)

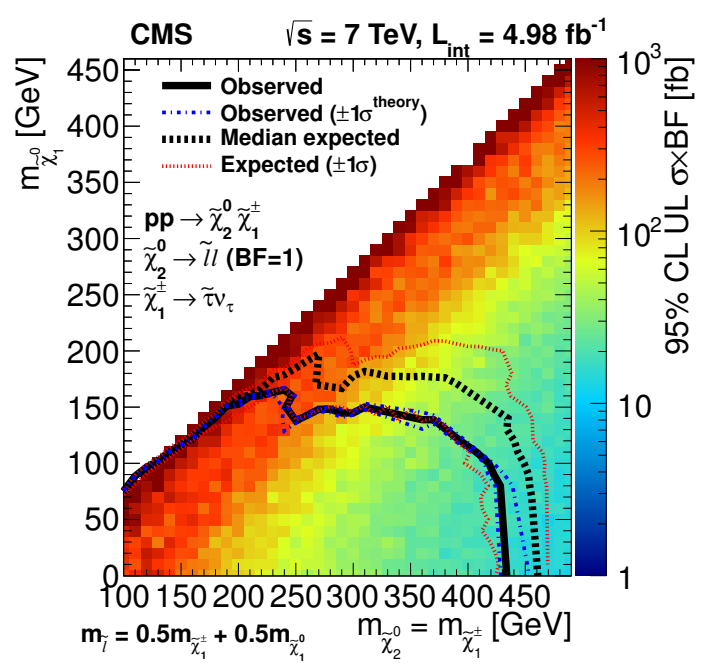

(b)

Figure 7. The shading in the $m_{\widetilde{\chi}_{1}^{0}}$ versus $m_{\widetilde{\chi}_{2}^{0}}\left(=m_{\widetilde{\chi}_{1}^{ \pm}}\right)$plane indicates the $95 \%$ CL upper limit on the chargino-neutralino NLO production cross section times branching fraction in (a) the flavordemocratic scenario, and (b) the $\tau$-enriched scenario, based on the results of the three-lepton $+E_{\mathrm{T}}^{\mathrm{miss}}$ search using the data of ref. [24]. The slepton mass is the mean of the $\widetilde{\chi}_{1}^{0}$ and $\widetilde{\chi}_{1}^{ \pm}$masses, i.e., $x_{\tilde{\ell}}=0.5$. In (a), the solid (dotted) contours bound the observed (expected) mass region excluded at $95 \%$ CL for a branching fraction of $50 \%$, as appropriate for the three-lepton decay products in the flavor-democratic scenario. In (b), the same contours are for a branching fraction of 100\%, as appropriate for the $\tau$-enriched scenario, in which the final-state lepton from the chargino decay is always the $\tau$ lepton.

\subsection{Limits on SMS from the search with three leptons, $M_{\ell \ell}$, and $M_{\mathrm{T}}$, and from same-sign dilepton searches}

Figure 8 displays, for three values of $x_{\tilde{\ell}}$, the $95 \%$ CL upper limit on the chargino-neutralino production cross section times branching fraction in the flavor-democratic scenario, derived from the results of the three-lepton search using $M_{\mathrm{T}}$ and $M_{\ell \ell}$ and those of the SS dilepton search. The contours bound the mass regions excluded at 95\% CL for a branching fraction of $50 \%$, as appropriate for the visible decay products in this scenario. The contours based on the observations are shown for the separate searches and for the combination. This search has slightly better sensitivity than the complementary search based on the $E_{\mathrm{T}}^{\text {miss }}$ shape (figure 7) in the region where the difference between $m_{\widetilde{\chi}_{2}^{0}}=m_{\widetilde{\chi}_{1}^{ \pm}}$and $m_{\widetilde{\chi}_{1}^{0}}$ is small, and slightly worse sensitivity where this mass difference is large.

Figure 9 presents the corresponding limits for the $\tau$-enriched scenario. As the SS dilepton search does not have sensitivity for $x_{\tilde{\ell}}=0.50$, there is no limit curve for this search in figure 9(b). In the other limit curves in both figures 8 and 9, the increase in the combined mass limit from incorporation of the SS dilepton search ranges up to approximately $20 \mathrm{GeV}$.

Appendix A provides a prescription for emulating the event selection efficiency for this signature, in order to facilitate further interpretation of the results in electroweak SUSY production scenarios beyond the models considered in this paper. 


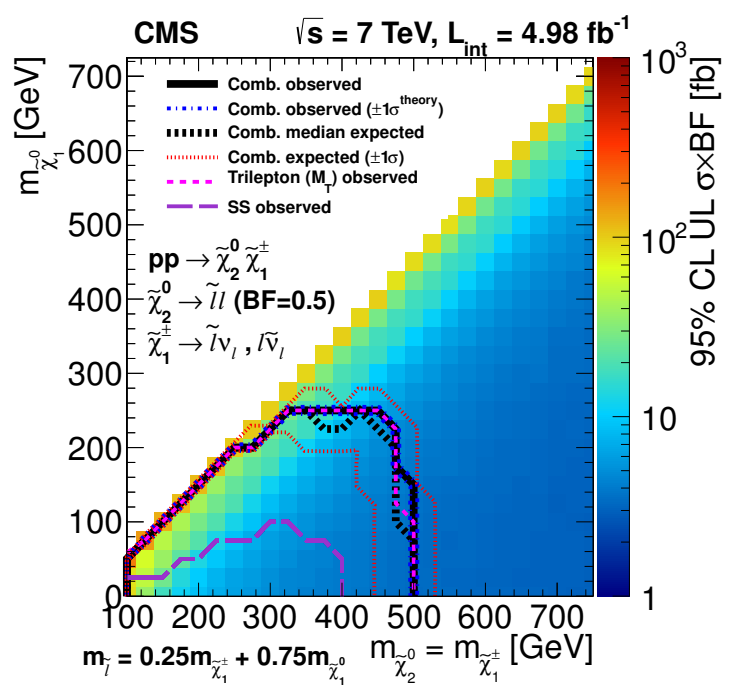

(a)

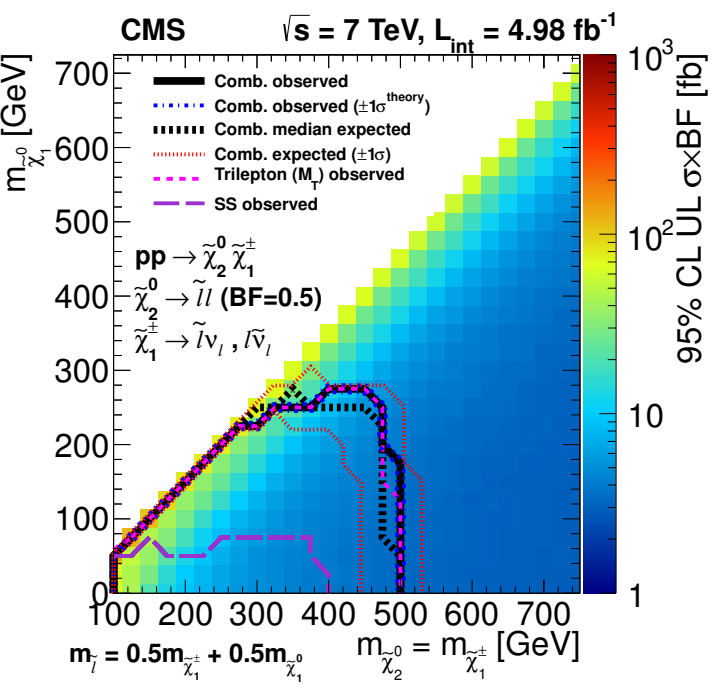

(b)

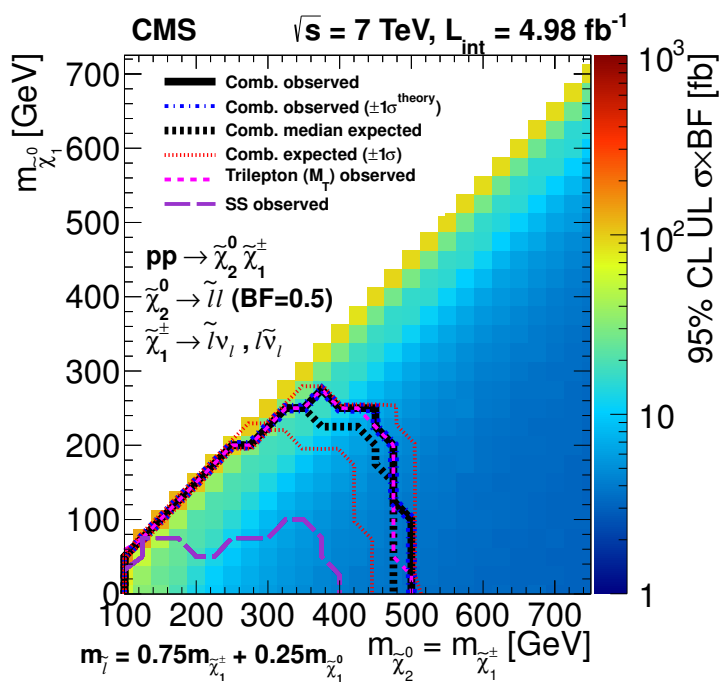

(c)

Figure 8. The shading in the $m_{\widetilde{\chi}_{1}^{0}}$ versus $m_{\widetilde{\chi}_{2}^{0}}\left(=m_{\widetilde{\chi}_{1}^{ \pm}}\right)$plane indicates the 95\% CL upper limit on the chargino-neutralino production NLO cross section times branching fraction in the flavordemocratic scenario, for the combined analysis of the three-lepton search using $M_{\ell \ell}$ and $M_{\mathrm{T}}$, and the same-sign dilepton search. The contours bound the mass regions excluded at 95\% CL for a branching fraction of $50 \%$, as appropriate for the visible decay products in this scenario. The contours based on the observations are shown for the separate searches and for the combination; in addition, the expected combined bound is shown. The three subfigures are the results for $x_{\tilde{\ell}}$ set to (a) 0.25 , (b) 0.50 , and (c) 0.75 . 


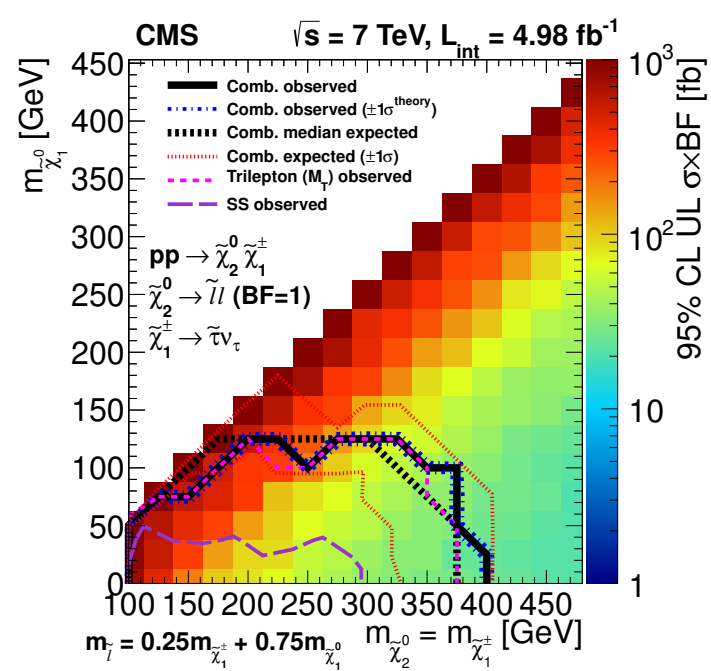

(a)

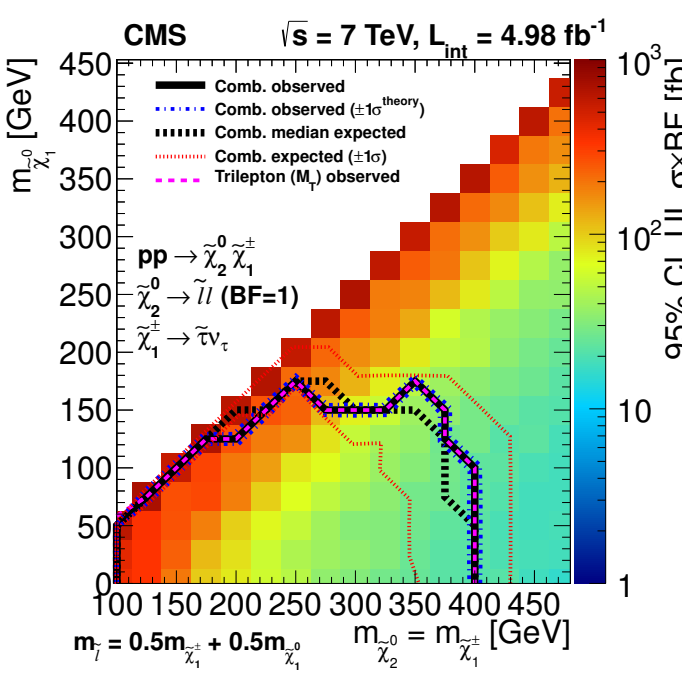

(b)

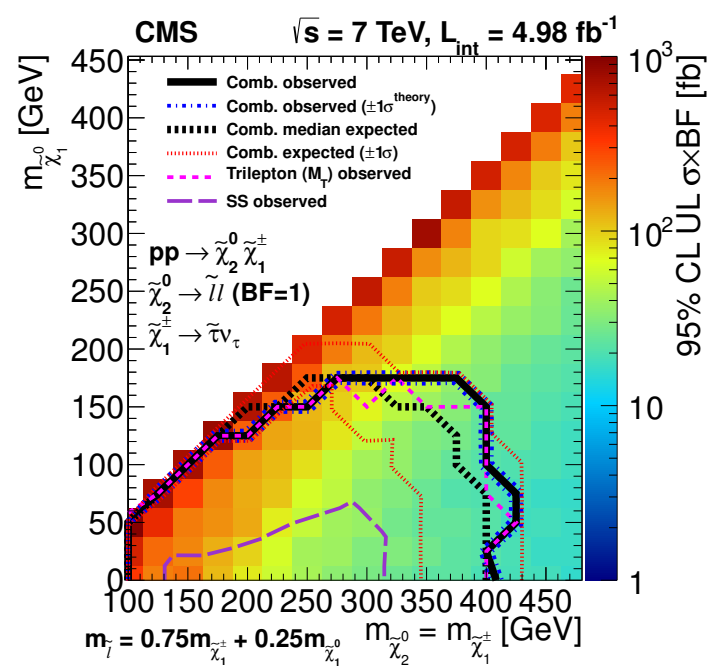

(c)

Figure 9. For the $\tau$-enriched scenario, the results corresponding to those in figure 8 .

\subsection{Limits on SMS and GMSB from the $\mathrm{WZ} / \mathrm{ZZ}+E_{\mathrm{T}}^{\text {miss }}$ final state with two or more leptons}

We calculate upper limits on the cross sections for pair production of charginos and neutralinos times branching fractions into the $\mathrm{WZ}+E_{\mathrm{T}}^{\mathrm{miss}}$ and $\mathrm{ZZ}+E_{\mathrm{T}}^{\text {miss }}$ final states as a function of the chargino and neutralino masses. In calculating these limits, the uncertainties related to jet and $E_{\mathrm{T}}^{\text {miss }}$ quantities (jet multiplicity, dijet mass, and $E_{\mathrm{T}}^{\text {miss }}$ ) vary significantly across the model space, and are addressed separately at each point, taking into account the bin-to-bin migration of signal events. The limits in section 6.3.1 are presented in the context of the SMS of figure 2(a) with $100 \%$ branching fractions of the chargino (neutralino) to $\mathrm{W}+\widetilde{\chi}_{1}^{0}\left(\mathrm{Z}+\widetilde{\chi}_{1}^{0}\right)$. The wino-like cross section with coupling $g \gamma^{\mu}$ 
is assumed. As the present data do not have sufficient sensitivity to explore the SMS of figure 2(b), the limits in section 6.3 .2 are presented in the context of a gauge-mediated symmetry breaking (GMSB) Z-enriched higgsino model [35-37] that has a large branching fraction to the $\mathrm{ZZ}+E_{\mathrm{T}}^{\text {miss }}$ final state. In this scenario, the LSP is a very light gravitino $($ mass $\leq 1 \mathrm{keV})$.

\subsubsection{Limits on SMS with on-shell $\mathrm{W}$ and $\mathrm{Z}$ from $\mathrm{WZ}+E_{\mathrm{T}}^{\text {miss }}$ and three-lepton analyses}

For limits on the SMS of figure 2(a) with on-shell $\mathrm{W}$ and $\mathrm{Z}$ bosons, we combine the results of the $\mathrm{WZ} / \mathrm{ZZ}+E_{\mathrm{T}}^{\text {miss }}$ analysis and the three-lepton analysis of section 3.2. From the $\mathrm{WZ} / \mathrm{ZZ}+E_{\mathrm{T}}^{\mathrm{miss}}$ analysis, we use the results in exclusive $E_{\mathrm{T}}^{\text {miss }}$ regions, as summarized in table 5. For the three-lepton analysis, we use the results in table 3. The three-lepton region with the broadest sensitivity is Region III, the on-Z, high- $M_{\mathrm{T}}$ region. If the difference between the common mass $m_{\widetilde{\chi}_{2}^{0}}=m_{\widetilde{\chi}_{1}^{ \pm}}$and $m_{\widetilde{\chi}_{1}^{0}}$ is small, then a significant fraction of the signal events fall below the $\mathrm{Z}$ mass window so that other signal regions contribute as well, in particular Region I (below-Z, low- $M_{\mathrm{T}}$ region). Region $\mathrm{VI}$ is not used directly in the fit, in order to facilitate the combination and to avoid using this region to constrain the WZ yield in the $\mathrm{WZ} / \mathrm{ZZ}+E_{\mathrm{T}}^{\mathrm{miss}}$ analysis, where the kinematic selection is very different since it includes jet requirements. Instead, a scaling factor of $1.1 \pm 0.1$ is applied to the WZ yield in Regions I-V, based on the data/simulation comparison in Region VI.

In the combination, the common signal-related systematic uncertainties for luminosity, jet energy scale, lepton identification, trigger efficiency, and misidentification of light-flavor jets as $\mathrm{b}$ jets are considered to be $100 \%$ correlated. For backgrounds, the only common systematic uncertainty is that for the WZ/ZZ simulation, which is treated as $100 \%$ correlated. No events in the data pass both signal selections. For the backgrounds, the overlap in the control sample is less than $1 \%$. Thus the two selections are treated as independent.

Figure 10 displays the observed limits for the two individual analyses and the combination. For large $m_{\widetilde{\chi}_{2}^{0}}=m_{\widetilde{\chi}_{1}^{ \pm}}$, the $\mathrm{WZ} / \mathrm{ZZ}+E_{\mathrm{T}}^{\text {miss }}$ analysis has higher sensitivity due to the large hadronic branching fractions of the $\mathrm{W}$ and $\mathrm{Z}$ bosons. At lower $m_{\widetilde{\chi}_{2}^{0}}=m_{\widetilde{\chi}_{1}^{ \pm}}$, the signal events do not have large $E_{\mathrm{T}}^{\text {miss }}$, resulting in a loss of signal region acceptance for the $\mathrm{WZ} / \mathrm{ZZ}+E_{\mathrm{T}}^{\mathrm{miss}}$ analysis. In this region, the background suppression provided by the requirement of a third lepton leads to better sensitivity for the three-lepton analysis.

\subsubsection{Limits on a Z-enriched GMSB model from $\mathrm{ZZ}+E_{\mathrm{T}}^{\text {miss }}$ and four-lepton search}

For the SMS of figure 2(b) with two on-shell $\mathrm{Z}$ bosons, the present data do not exclude any region of $m_{\widetilde{\chi}_{2}^{0}}=m_{\widetilde{\chi}_{1}^{ \pm}}$, and are therefore not sensitive to a scenario in which neutralino pair production is the sole production mechanism. However, the $\mathrm{ZZ}+E_{\mathrm{T}}^{\mathrm{miss}}$ signature can be enhanced in scenarios in which additional mechanisms, such as chargino-chargino and chargino-neutralino production, also contribute. This is the case in a GMSB Z-enriched higgsino model [35-37].

In this scenario, the LSP is a nearly massless gravitino, the next-to-lightest SUSY particle is a Z-enriched higgsino $\widetilde{\chi}_{1}^{0}$, and the $\widetilde{\chi}_{1}^{ \pm}$is nearly mass degenerate with the $\widetilde{\chi}_{1}^{0}$. 


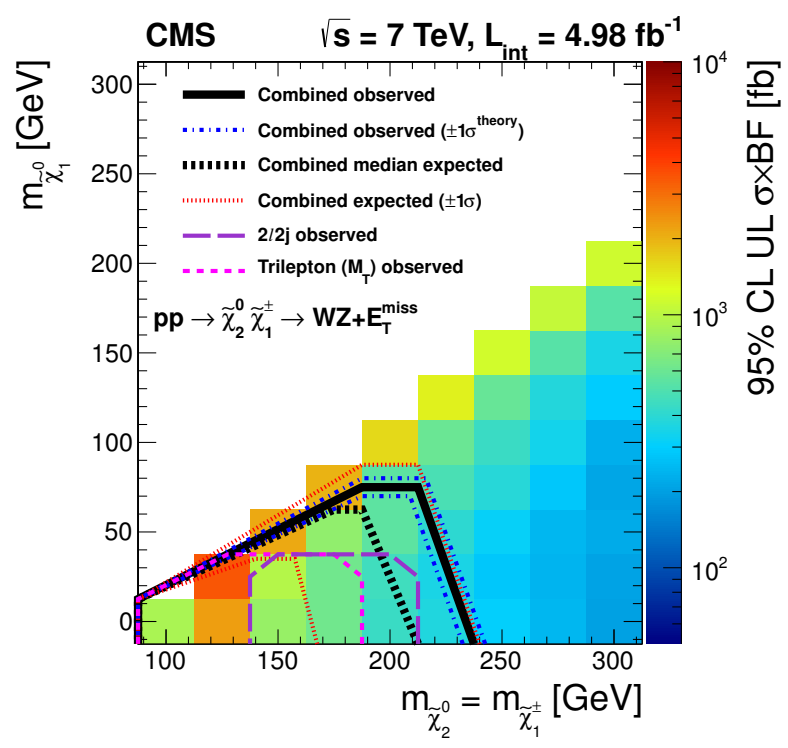

Figure 10. Interpretation of the $\mathrm{WZ}+E_{\mathrm{T}}^{\mathrm{miss}}$ and three-lepton results in the context of the $\mathrm{WZ}$ SMS. The $\mathrm{WZ}+E_{\mathrm{T}}^{\text {miss }}$ observed, three-lepton observed, combined observed, and combined expected contours are indicated.

We set the gaugino mass parameters $M_{1}$ and $M_{2}$ to $M_{1}=M_{2}=1 \mathrm{TeV}$, the ratio of Higgs expectation values $\tan \beta$ to $\tan \beta=2$, and then explore variable Higgsino mass parameters. The masses of the $\widetilde{\chi}_{1}^{0}$ and $\widetilde{\chi}_{1}^{ \pm}$are controlled by the parameter $\mu$, with $m_{\widetilde{\chi}_{1}^{0}} \approx$ $m_{\widetilde{\chi}_{1}^{ \pm}} \approx \mu$. Hence the $\widetilde{\chi}_{1}^{ \pm}$decays to $\widetilde{\chi}_{1}^{0}$ and to low- $p_{\mathrm{T}}$ SM particles that escape detection. Thus, all production mechanisms (chargino-chargino, chargino-neutralino, and neutralinoneutralino) lead to a pair of $\widetilde{\chi}_{1}^{0}$ particles in the final state, and the branching fraction to the $\mathrm{ZZ}+E_{\mathrm{T}}^{\mathrm{miss}}$ final state is large (varying from $100 \%$ at $\mu=130 \mathrm{GeV}$ to $85 \%$ at $\mu=410 \mathrm{GeV}$ ). Mainly because of the mix of production mechanisms, the kinematic distributions of this model are slightly different than those expected in a pure neutralinopair production scenario, leading to different signal acceptances.

We combine the results of the $\mathrm{WZ} / \mathrm{ZZ}+E_{\mathrm{T}}^{\text {miss }}$ analysis of section 5 with independent results for the four-lepton channel analysis of ref. [24] to further restrict the GMSB scenario. The two selections have negligible overlap, and are thus treated as independent in the combination.

Table 6 summarizes the relevant results from ref. [24], with the high- $H_{\mathrm{T}}$ and low- $H_{\mathrm{T}}$ regions of that study combined. All samples contain four leptons, including an OSSF lepton pair whose mass is consistent with the $\mathrm{Z}$ boson mass, with separate entries for events with $E_{\mathrm{T}}^{\text {miss }}$ above or below $50 \mathrm{GeV}$, and for events with zero or one hadronically decaying $\tau$ lepton candidate $\left(\tau_{h}\right)$.

The results of the individual and combined exclusions are displayed in figure 11. As in section 6.3.1, the $\mathrm{WZ} / \mathrm{ZZ}+E_{\mathrm{T}}^{\text {miss }}$ and the multilepton analysis are complementary, with the four-lepton analysis having greater (less) sensitivity than the $\mathrm{WZ} / \mathrm{ZZ}+E_{\mathrm{T}}^{\mathrm{miss}}$ analysis at small (large) values of $\mu$. By combining the two analyses, we exclude the range of $\mu$ between 148 and $248 \mathrm{GeV}$. 


\begin{tabular}{|lcc|}
\hline Signal Region & Expected Background & Observed Yield \\
\hline$N\left(\tau_{h}\right)=0, E_{\mathrm{T}}^{\text {miss }} \geq 50 \mathrm{GeV}$ & $1.0 \pm 0.2$ & 1 \\
$N\left(\tau_{h}\right)=0, E_{\mathrm{T}}^{\text {miss }}<50 \mathrm{GeV}$ & $38 \pm 15$ & 34 \\
$N\left(\tau_{h}\right)=1, E_{\mathrm{T}}^{\text {miss }} \geq 50 \mathrm{GeV}$ & $2.6 \pm 0.7$ & 4 \\
$N\left(\tau_{h}\right)=1, E_{\mathrm{T}}^{\text {miss }}<50 \mathrm{GeV}$ & $18.0 \pm 5.2$ & 20 \\
\hline
\end{tabular}

Table 6. Summary of the results from the multilepton analysis of ref. [24] used as input to the combined limit on the GMSB model. All categories have four leptons including an OSSF pair consistent with a $\mathrm{Z}$ boson; $N\left(\tau_{h}\right)$ denotes the number of these leptons that are identified as hadronically decaying $\tau$ leptons. Uncertainties include statistical and systematic contributions.

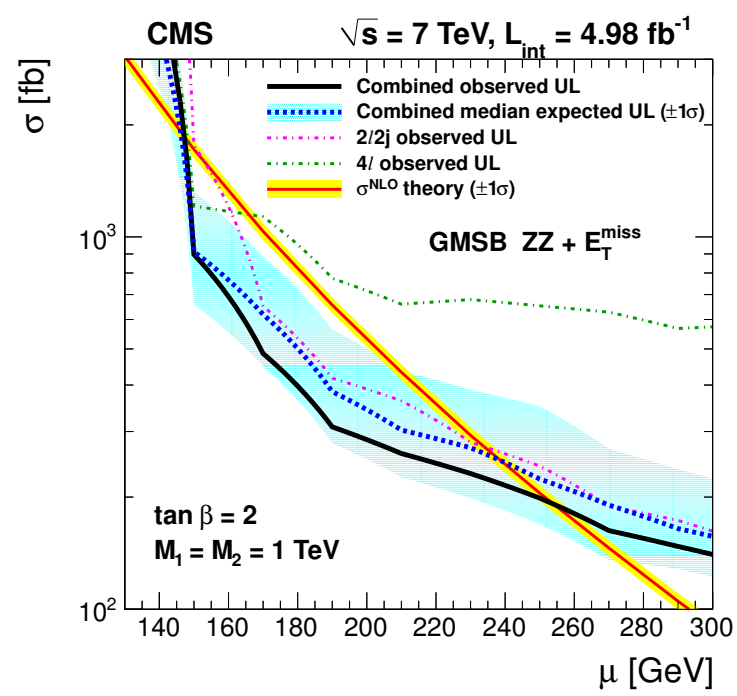

Figure 11. Interpretation of the results for the $\mathrm{ZZ}+E_{\mathrm{T}}^{\text {miss }}$ (with two leptons and two jets) analysis and the results of the four-lepton analysis from ref. [24] in the context of the GMSB model described in the text. The NLO cross section upper limits are presented for the $\mathrm{ZZ}+E_{\mathrm{T}}^{\mathrm{miss}}$ observed, multilepton observed, the combined observed, and the combined expected results. The theory prediction for the cross section is also presented. The median expected limits, their $\pm 1 \sigma$ variations, and the $\pm 1 \sigma$ band on the theory curve are as described at the beginning of section 6 .

\subsection{Summary of excluded masses for chargino-neutralino pair production}

Figure 12 displays a summary of the excluded regions in the chargino-neutralino production scenarios considered above. Also displayed are the exclusion curves at 95\% CL from searches at LEP2 $[16,17,70]$, which excluded $m_{\tilde{\ell}}<82 \mathrm{GeV}$ and $m_{\tilde{\chi}_{1}^{ \pm}}<103 \mathrm{GeV}$. The results in this paper probe the production of charginos and neutralinos with masses up to approximately 200 to $500 \mathrm{GeV}$, depending on the decay modes of these particles.

\section{Summary}

This paper presents searches for supersymmetric charginos and neutralinos. While a number of previous studies at the LHC have focused on strongly coupled supersymmetric particles, this paper is one of the first to focus on the electroweak sector of supersymmetry. 


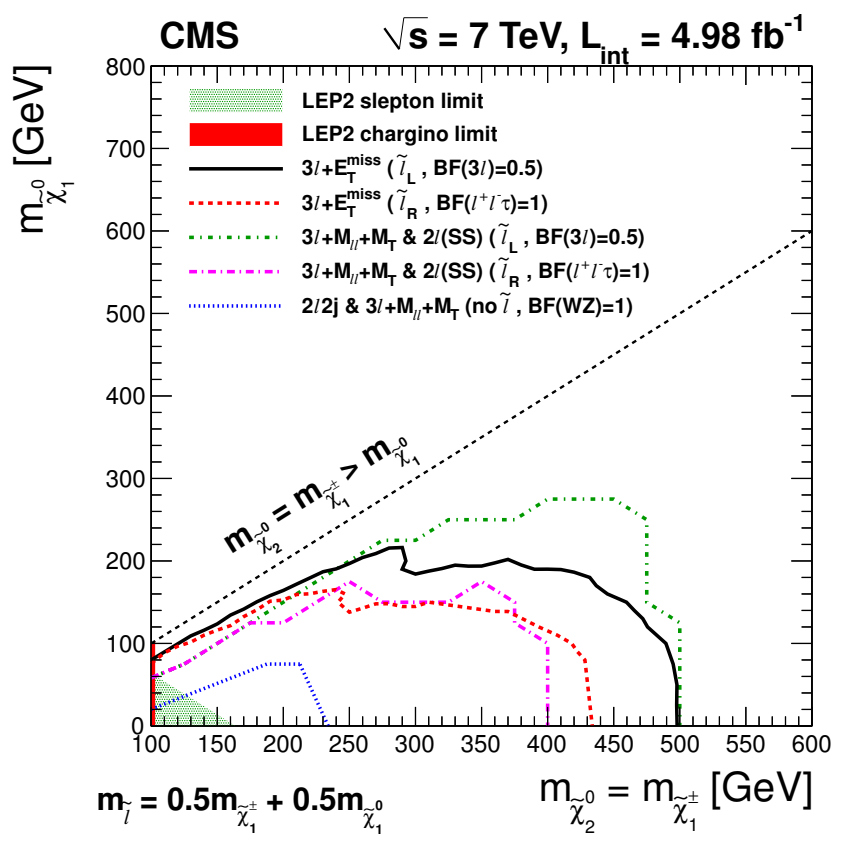

Figure 12. Summary of the excluded regions in the $m_{\widetilde{\chi}_{1}^{0}}$ versus $m_{\widetilde{\chi}_{2}^{0}}\left(=m_{\widetilde{\chi}_{1}^{ \pm}}\right)$plane for: the threelepton $+E_{\mathrm{T}}^{\mathrm{miss}}$ search (sections 3.1 and 6.1 ), separately for the $\widetilde{\ell}_{L}$ and $\widetilde{\ell}_{R}$ scenarios; the combination (section 6.2) of the three-lepton analysis based on $M_{\ell \ell}$ and $M_{\mathrm{T}}$ (section 3.2) with the SS dilepton analysis (section 4), separately for the $\tilde{\ell}_{L}$ and $\widetilde{\ell}_{R}$ scenarios; and the combination (section 6.3.1) of the diboson analysis with two leptons and two jets (section 5) with the three-lepton analysis based on $M_{\ell \ell}$ and $M_{\mathrm{T}}$ (section 3.2), for the $\mathrm{WZ}+E_{\mathrm{T}}^{\mathrm{miss}}$ model. Regions excluded by searches at LEP2 for sleptons and charginos are also indicated. The implied branching fractions introduced in section 1 are noted in the legend. For models with intermediate sleptons (including the LEP2 slepton limit), the interpretations correspond to $x_{\widetilde{\ell}}=0.5$.

The searches performed here explore final states with exactly three leptons using transverse mass and lepton-pair invariant mass, two same-sign leptons, and two opposite-sign leptons and two jets. The results of a published search for new physics in the final state of three or more leptons are reinterpreted in the context of electroweak supersymmetry. No excesses above the standard model expectations are observed. The results are used to exclude a range of chargino and neutralino masses from approximately 200 to $500 \mathrm{GeV}$ in the context of models that assume large branching fractions of charginos and neutralinos to leptons and vector bosons.

\section{Acknowledgments}

We thank David Shih for useful discussions and help with implementation of the Z-enriched GMSB model of section 6.3.2.

We congratulate our colleagues in the CERN accelerator departments for the excellent performance of the LHC machine. We thank the technical and administrative staff at CERN and other CMS institutes. This work was supported by the Austrian Federal 
Ministry of Science and Research; the Belgian Fonds de la Recherche Scientifique, and Fonds voor Wetenschappelijk Onderzoek; the Brazilian Funding Agencies (CNPq, CAPES, FAPERJ, and FAPESP); the Bulgarian Ministry of Education and Science; CERN; the Chinese Academy of Sciences, Ministry of Science and Technology, and National Natural Science Foundation of China; the Colombian Funding Agency (COLCIENCIAS); the Croatian Ministry of Science, Education and Sport; the Research Promotion Foundation, Cyprus; the Ministry of Education and Research, Recurrent financing contract SF0690030s09 and European Regional Development Fund, Estonia; the Academy of Finland, Finnish Ministry of Education and Culture, and Helsinki Institute of Physics; the Institut National de Physique Nucléaire et de Physique des Particules / CNRS, and Commissariat à l'Énergie Atomique et aux Énergies Alternatives / CEA, France; the Bundesministerium für Bildung und Forschung, Deutsche Forschungsgemeinschaft, and Helmholtz-Gemeinschaft Deutscher Forschungszentren, Germany; the General Secretariat for Research and Technology, Greece; the National Scientific Research Foundation, and National Office for Research and Technology, Hungary; the Department of Atomic Energy and the Department of Science and Technology, India; the Institute for Studies in Theoretical Physics and Mathematics, Iran; the Science Foundation, Ireland; the Istituto Nazionale di Fisica Nucleare, Italy; the Korean Ministry of Education, Science and Technology and the World Class University program of NRF, Korea; the Lithuanian Academy of Sciences; the Mexican Funding Agencies (CINVESTAV, CONACYT, SEP, and UASLP-FAI); the Ministry of Science and Innovation, New Zealand; the Pakistan Atomic Energy Commission; the Ministry of Science and Higher Education and the National Science Centre, Poland; the Fundação para a Ciência e a Tecnologia, Portugal; JINR (Armenia, Belarus, Georgia, Ukraine, Uzbekistan); the Ministry of Education and Science of the Russian Federation, the Federal Agency of Atomic Energy of the Russian Federation, Russian Academy of Sciences, and the Russian Foundation for Basic Research; the Ministry of Science and Technological Development of Serbia; the Secretaría de Estado de Investigación, Desarrollo e Innovación and Programa Consolider-Ingenio 2010, Spain; the Swiss Funding Agencies (ETH Board, ETH Zurich, PSI, SNF, UniZH, Canton Zurich, and SER); the National Science Council, Taipei; the Thailand Center of Excellence in Physics, the Institute for the Promotion of Teaching Science and Technology and National Electronics and Computer Technology Center; the Scientific and Technical Research Council of Turkey, and Turkish Atomic Energy Authority; the Science and Technology Facilities Council, UK; the US Department of Energy, and the US National Science Foundation.

Individuals have received support from the Marie-Curie programme and the European Research Council (European Union); the Leventis Foundation; the A. P. Sloan Foundation; the Alexander von Humboldt Foundation; the Austrian Science Fund (FWF); the Belgian Federal Science Policy Office; the Fonds pour la Formation à la Recherche dans l'Industrie et dans l'Agriculture (FRIA-Belgium); the Agentschap voor Innovatie door Wetenschap en Technologie (IWT-Belgium); the Ministry of Education, Youth and Sports (MEYS) of Czech Republic; the Council of Science and Industrial Research, India; the Compagnia di San Paolo (Torino); and the HOMING PLUS programme of Foundation for Polish Science, cofinanced from European Union, Regional Development Fund. 


\begin{tabular}{|lccccccc|}
\hline$x$ & $p_{0}$ & $p_{1}$ & $p_{2}$ & $p_{3}$ & $p_{4}$ & $p_{5}$ & $p_{6}$ \\
\hline$p_{\mathrm{T}}(\mu)$ & -4.65 & 27.38 & -14.64 & -9.31 & 0.47 & -849.3 & 0. \\
$p_{\mathrm{T}}(\mathrm{e})$, & 12.32 & 10.11 & 20.12 & 32.17 & 0.32 & 0.11 & 0. \\
$E_{\mathrm{T}}^{\text {miss }}$ & 48.37 & 43.54 & 49.90 & 14.95 & 0.06 & 0.44 & 0. \\
$M_{\mathrm{T}}$ & 98.23 & 87.99 & 97.61 & 29.78 & 0.36 & 0.14 & 0.008 \\
$p_{\mathrm{T}}$ (b quark) & 30.60 & 31.80 & 0.34 & 0. & 0. & 0. & 0. \\
$p_{\mathrm{T}}$ (c quark) & 32.02 & 45.34 & 0.11 & 0. & 0. & 0. & 0. \\
$p_{\mathrm{T}}$ (udsg parton) & 68.84 & 55.21 & 0.02 & 0. & 0. & 0. & 0. \\
\hline
\end{tabular}

Table 7. The parameters of the efficiency function $\epsilon(x)$, where $x$ represents $p_{\mathrm{T}}(\mu), p_{\mathrm{T}}(\mathrm{e}), E_{\mathrm{T}}^{\text {miss }}$, $M_{\mathrm{T}}$, or $p_{\mathrm{T}}$ (parton) for different quark flavors (udscb) and for gluons (g).

\section{A Signal efficiency model for the three-lepton analysis with $M_{\ell \ell}$ and $M_{\mathrm{T}}$}

In order to facilitate the interpretation of the three-lepton results with $M_{\ell \ell}$ and $M_{\mathrm{T}}$ presented in section 3.2 within the context of other signal models that are not considered here, we provide a prescription for emulating the event selection efficiency. This prescription includes lepton reconstruction and identification efficiencies, $E_{\mathrm{T}}^{\text {miss }}$ and $M_{\mathrm{T}}$ selection efficiencies, as well as the b-jet identification probability. The latter can be used to parameterize the b-veto acceptance in case the model of interest contains such jets.

We perform a fit to efficiency curves for each selection using the parametric function

$$
\epsilon(x)=p_{6}+p_{4}\left[\operatorname{erf}\left(\frac{\mathrm{x}-\mathrm{p}_{0}}{\mathrm{p}_{1}}\right)+1\right]+p_{5}\left[\operatorname{erf}\left(\frac{\mathrm{x}-\mathrm{p}_{2}}{\mathrm{p}_{3}}\right)+1\right]
$$

where $x$ represents the observable for which the efficiency is parametrized, and erf indicates the error function. This includes the efficiency for electrons and muons to be reconstructed and to satisfy the identification requirements as a function of the lepton $p_{\mathrm{T}}$; the probability for an event to satisfy the requirements $E_{\mathrm{T}}^{\text {miss }}>50 \mathrm{GeV}$ and $M_{\mathrm{T}}>100 \mathrm{GeV}$ as a function of true $E_{\mathrm{T}}^{\mathrm{miss}}$ and true $M_{\mathrm{T}}$; and the probability for a jet to be identified as a b jet separately for the cases where the jet originates from a b-, c-, or light-flavor quark or gluon as a function of jet $p_{\mathrm{T}}$. (The true $E_{\mathrm{T}}^{\text {miss }}$ observable is calculated with the stable generator-level invisible particles, while the true $M_{\mathrm{T}}$ is calculated using the true $E_{\mathrm{T}}^{\text {miss }}$ and the third lepton, i.e., the one not used in the $M_{\ell \ell}$ calculation.)

The parameters of the fitted functions are given in table 7. Using these parameters and the values of $x$, a combined probability for a given event to pass the full event selection can be obtained. We have tested the efficiency model in a signal sample and observed consistent event yields compared to the full detector simulation within about $25 \%$.

Open Access. This article is distributed under the terms of the Creative Commons Attribution License which permits any use, distribution and reproduction in any medium, provided the original author(s) and source are credited. 


\section{References}

[1] Y. Golfand and E. Likhtman, Extension of the algebra of Poincaré group generators and violation of $p$ invariance, JETP Lett. 13 (1971) 323 [INSPIRE].

[2] P. Ramond, Dual theory for free fermions, Phys. Rev. D 3 (1971) 2415 [inSPIRE].

[3] A. Neveu and J. Schwarz, Factorizable dual model of pions, Nucl. Phys. B 31 (1971) 86 [INSPIRE].

[4] A. Neveu and J. Schwarz, Quark model of dual pions, Phys. Rev. D 4 (1971) 1109 [INSPIRE].

[5] D. Volkov and V. Akulov, Is the neutrino a Goldstone particle?, Phys. Lett. B 46 (1973) 109 [INSPIRE].

[6] J. Wess and B. Zumino, A lagrangian model invariant under supergauge transformations, Phys. Lett. B 49 (1974) 52 [INSPIRE].

[7] J. Wess and B. Zumino, Supergauge transformations in four-dimensions, Nucl. Phys. B 70 (1974) 39 [INSPIRE].

[8] D.A. Dicus, S. Nandi and X. Tata, W decay in supergravity GUTS, Phys. Lett. B 129 (1983) 451 [Erratum ibid. B 145 (1984) 448] [INSPIRE].

[9] A.H. Chamseddine, P. Nath and R.L. Arnowitt, Experimental signals for supersymmetric decays of the $W$ and $Z$ bosons, Phys. Lett. B 129 (1983) 445 [Erratum ibid. B 132 (1983) 467] [INSPIRE].

[10] H. Baer and X. Tata, Multi-lepton signals from $W^{ \pm}$and $Z^{0}$ decays to gauginos at $\bar{p} p$ colliders, Phys. Lett. B 155 (1985) 278 [inSPIRE].

[11] P. Nath and R.L. Arnowitt, Supersymmetric Signals at the Tevatron, Mod. Phys. Lett. A 2 (1987) 331 [INSPIRE].

[12] H. Baer, C.-h. Chen, F. Paige and X. Tata, Trileptons from chargino-neutralino production at the CERN Large Hadron Collider, Phys. Rev. D 50 (1994) 4508 [hep-ph/9404212] [INSPIRE].

[13] H. Baer, C.-h. Chen, F. Paige and X. Tata, Signals for minimal supergravity at the CERN Large Hadron Collider. 2: multi-lepton channels, Phys. Rev. D 53 (1996) 6241 [hep-ph/9512383] [INSPIRE].

[14] CMS collaboration, Absolute calibration of the luminosity measurement at CMS: winter 2012 update, PAS-SMP-12-008 (2012).

[15] ALEPH collaboration, A. Heister et al., Absolute mass lower limit for the lightest neutralino of the MSSM from $e^{+} e^{-}$data at $\sqrt{s}$ up to 209 GeV, Phys. Lett. B 583 (2004) 247 [InSPIRE].

[16] ALEPH collaboration, A. Heister et al., Absolute lower limits on the masses of selectrons and sneutrinos in the MSSM, Phys. Lett. B 544 (2002) 73 [hep-ex/0207056] [INSPIRE].

[17] DELPHI collaboration, J. Abdallah et al., Searches for supersymmetric particles in $e^{+} e^{-}$ collisions up to $208 \mathrm{GeV}$ and interpretation of the results within the MSSM, Eur. Phys. J. C 31 (2003) 421 [hep-ex/0311019] [INSPIRE].

[18] L3 collaboration, P. Achard et al., Search for scalar leptons and scalar quarks at LEP, Phys. Lett. B 580 (2004) 37 [hep-ex/0310007] [INSPIRE].

[19] OPAL collaboration, G. Abbiendi et al., Search for chargino and neutralino production at $\sqrt{s}=192$ GeV to 209 GeV at LEP, Eur. Phys. J. C 35 (2004) 1 [hep-ex/0401026] [INSPIRE]. 


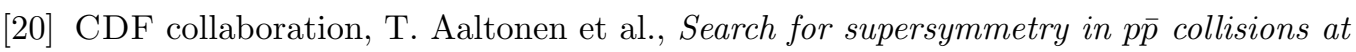
$\sqrt{s}=1.96 \mathrm{TeV}$ using the trilepton signature of chargino-neutralino production, Phys. Rev. Lett. 101 (2008) 251801 [arXiv:0808.2446] [INSPIRE].

[21] D0 collaboration, V. Abazov et al., Search for associated production of charginos and neutralinos in the trilepton final state using $2.3 \mathrm{fb}^{-1}$ of data, Phys. Lett. B 680 (2009) 34 [arXiv:0901.0646] [INSPIRE].

[22] ATLAS collaboration, G. Aad et al., Search for supersymmetry in events with three leptons and missing transverse momentum in $\sqrt{s}=7 \mathrm{TeV}$ pp collisions with the ATLAS detector, Phys. Rev. Lett. 108 (2012) 261804 [arXiv:1204.5638] [inSPIRE].

[23] CMS collaboration, S. Chatrchyan et al., Search for physics beyond the standard model using multilepton signatures in pp collisions at $\sqrt{s}=7$ TeV, Phys. Lett. B 704 (2011) 411 [arXiv:1106.0933] [INSPIRE].

[24] CMS collaboration, S. Chatrchyan et al., Search for anomalous production of multilepton events in pp collisions at $\sqrt{s}=7 \mathrm{TeV}$, JHEP 06 (2012) 169 [arXiv:1204.5341] [INSPIRE].

[25] CMS collaboration, S. Chatrchyan et al., Search for physics beyond the standard model in events with a $Z$ boson, jets and missing transverse energy in pp collisions at $\sqrt{s}=7 \mathrm{TeV}$, Phys. Lett. B 716 (2012) 260 [arXiv:1204.3774] [InSPIRE].

[26] CMS collaboration, S. Chatrchyan et al., Search for new physics with same-sign isolated dilepton events with jets and missing transverse energy, Phys. Rev. Lett. 109 (2012) 071803 [arXiv:1205.6615] [INSPIRE].

[27] B. Knuteson and S. Mrenna, BARD: interpreting new frontier energy collider physics, hep-ph/0602101 [INSPIRE].

[28] N. Arkani-Hamed et al., MARMOSET: the path from LHC data to the new standard model via on-shell effective theories, hep-ph/0703088 [INSPIRE].

[29] S. Dube, J. Glatzer, S. Somalwar, A. Sood and S. Thomas, Addressing the multi-channel inverse problem at high energy colliders: a model independent approach to the search for new physics with trileptons, J. Phys. G 39 (2012) 085004 [arXiv:0808.1605] [INSPIRE].

[30] J. Alwall, P. Schuster and N. Toro, Simplified models for a first characterization of new physics at the LHC, Phys. Rev. D 79 (2009) 075020 [arXiv:0810.3921] [InSPIRE].

[31] J. Alwall, M.-P. Le, M. Lisanti and J.G. Wacker, Model-independent jets plus missing energy searches, Phys. Rev. D 79 (2009) 015005 [arXiv: 0809.3264] [InSPIRE].

[32] LHC New Physics Working Group collaboration, D. Alves et al., Simplified models for LHC new physics searches, J. Phys. G 39 (2012) 105005 [arXiv:1105.2838] [INSPIRE].

[33] D.S. Alves, E. Izaguirre and J.G. Wacker, Where the sidewalk ends: jets and missing energy search strategies for the $7 \mathrm{TeV}$ LHC, JHEP 10 (2011) 012 [arXiv:1102.5338] [INSPIRE].

[34] M. Papucci, J.T. Ruderman and A. Weiler, Natural SUSY endures, JHEP 09 (2012) 035 [arXiv: 1110.6926] [INSPIRE].

[35] K.T. Matchev and S.D. Thomas, Higgs and $Z$ boson signatures of supersymmetry, Phys. Rev. D 62 (2000) 077702 [hep-ph/9908482] [INSPIRE].

[36] P. Meade, M. Reece and D. Shih, Prompt decays of general neutralino NLSPs at the Tevatron, JHEP 05 (2010) 105 [arXiv:0911.4130] [INSPIRE]. 
[37] J.T. Ruderman and D. Shih, General neutralino NLSPs at the early LHC, JHEP 08 (2012) 159 [arXiv: 1103.6083] [INSPIRE].

[38] K.T. Matchev and D.M. Pierce, Supersymmetry reach of the Tevatron via trilepton, like sign dilepton and dilepton plus $\tau$ jet signatures, Phys. Rev. D 60 (1999) 075004 [hep-ph/9904282] [INSPIRE].

[39] H. Baer, M. Drees, F. Paige, P. Quintana and X. Tata, Trilepton signal for supersymmetry at the Fermilab Tevatron revisited, Phys. Rev. D 61 (2000) 095007 [hep-ph/9906233] [inSPIRE].

[40] K.T. Matchev and D.M. Pierce, New backgrounds in trilepton, dilepton and dilepton plus $\tau$ jet SUSY signals at the Tevatron, Phys. Lett. B 467 (1999) 225 [hep-ph/9907505] [INSPIRE].

[41] V.D. Barger and C. Kao, Trilepton signature of minimal supergravity at the upgraded Tevatron, Phys. Rev. D 60 (1999) 115015 [hep-ph/9811489] [INSPIRE].

[42] ATLAS collaboration, G. Aad et al., Search for direct production of charginos and neutralinos in events with three leptons and missing transverse momentum in $\sqrt{s}=7$ TeV pp collisions with the ATLAS detector, arXiv:1208.3144 [INSPIRE].

[43] ATLAS collaboration, G. Aad et al., Search for direct slepton and gaugino production in final states with two leptons and missing transverse momentum with the ATLAS detector in pp collisions at $\sqrt{s}=7 \mathrm{TeV}$, arXiv:1208.2884 [INSPIRE].

[44] CMS collaboration, S. Chatrchyan et al., The CMS experiment at the CERN LHC, 2008 JINST 3 S08004 [INSPIRE].

[45] F. Maltoni and T. Stelzer, MadEvent: automatic event generation with MadGraph, JHEP 02 (2003) 027 [hep-ph/0208156] [INSPIRE].

[46] J. Alwall, M. Herquet, F. Maltoni, O. Mattelaer and T. Stelzer, MadGraph 5: going beyond, JHEP 06 (2011) 128 [arXiv: 1106.0522] [INSPIRE].

[47] T. Sjöstrand, S. Mrenna and P.Z. Skands, A brief introduction to PYTHIA 8.1, Comput. Phys. Commun. 178 (2008) 852 [arXiv:0710.3820] [INSPIRE].

[48] P.M. Nadolsky et al., Implications of CTEQ global analysis for collider observables, Phys. Rev. D 78 (2008) 013004 [arXiv: 0802.0007] [INSPIRE].

[49] J.M. Campbell, R.K. Ellis and C. Williams, Vector boson pair production at the LHC, JHEP 07 (2011) 018 [arXiv: 1105.0020] [INSPIRE].

[50] W. Beenakker et al., Production of charginos, neutralinos, and sleptons at hadron colliders, Phys. Rev. Lett. 83 (1999) 3780 [Erratum 100 (2008) 029901] [hep-ph/9906298] [INSPIRE].

[51] M. Krämer et al., Supersymmetry production cross sections in pp collisions at $\sqrt{s}=7 \mathrm{TeV}$, arXiv:1206.2892 [INSPIRE].

[52] P.Z. Skands et al., SUSY Les Houches accord: Interfacing SUSY spectrum calculators, decay packages and event generators, JHEP 07 (2004) 036 [hep-ph/0311123] [INSPIRE].

[53] H. Baer, F.E. Paige, S.D. Protopopescu and X. Tata, Simulating supersymmetry with ISAJET \%.0/ISASUSY 1.0, hep-ph/9305342 [INSPIRE].

[54] W. Beenakker, R. Hopker and M. Spira, PROSPINO: a program for the production of supersymmetric particles in next-to-leading order QCD, hep-ph/9611232 [INSPIRE].

[55] CMS collaboration, The fast simulation of the CMS detector at the LHC, J. Phys. Conf. Ser. 331 (2011) 032049. 
[56] GEANT4 collaboration, S. Agostinelli et al., GEANT4 - A simulation toolkit, Nucl. Instrum. Meth. A 506 (2003) 250 [InSPIRE].

[57] CMS collaboration, Study of $\tau$ reconstruction algorithms using pp collisions data collected at $\sqrt{s}=7$ TeV, PAS-PFT-10-004 (2010).

[58] CMS collaboration, CMS Strategies for $\tau$ reconstruction and identification using particle-flow techniques, PAS-PFT-08-001 (2008).

[59] CMS collaboration, Electron reconstruction and identification at $\sqrt{s}=7$ TeV, PAS-EGM-10-004 (2010).

[60] CMS collaboration, S. Chatrchyan et al., Performance of CMS muon reconstruction in $\mathrm{pp}$ collision events at $\sqrt{s}=7$ TeV, 2012 JINST 7 P10002 [arXiv:1206.4071] [INSPIRE].

[61] CMS collaboration, Performance of $\tau$-lepton reconstruction and identification in CMS, 2012 JINST 7 P01001 [arXiv:1109.6034] [INSPIRE].

[62] M. Cacciari, G.P. Salam and G. Soyez, The anti- $k_{t}$ jet clustering algorithm, JHEP 04 (2008) 063 [arXiv:0802.1189] [INSPIRE].

[63] CMS collaboration, Performance of the b-jet identification in CMS, PAS-BTV-11-001 (2011).

[64] CMS collaboration, S. Chatrchyan et al., Measurement of the inclusive $W$ and $Z$ production cross sections in pp collisions at $\sqrt{s}=7$ TeV, JHEP 10 (2011) 132 [arXiv:1107.4789] [INSPIRE].

[65] J. Nachtman, D. Saltzberg and M. Worcester, Study of a like sign dilepton search for chargino neutralino production at CDF, FERMILAB-CONF-99-023-E (1999) [hep-ex/9902010].

[66] J.D. Lykken and K.T. Matchev, Supersymmetry signatures with $\tau$ jets at the Tevatron, Phys. Rev. D 61 (2000) 015001 [hep-ph/9903238] [INSPIRE].

[67] T. Junk, Confidence level computation for combining searches with small statistics, Nucl. Instrum. Meth. A 434 (1999) 435 [hep-ex/9902006] [InSPIRE].

[68] A.L. Read, Presentation of search results: the CL(s) technique, J. Phys. G 28 (2002) 2693 [INSPIRE].

[69] ATLAS and CMS collaborations, Procedure for the LHC Higgs boson search combination in summer 2011, CMS-NOTE-2011-005, ATL-PHYS-PUB-2011-11 (2011).

[70] Particle Data Group, J. Beringer et al., Review of particle physics, Phys. Rev. D 86 (2012) 010001 [INSPIRE]. 


\section{The CMS collaboration}

\section{Yerevan Physics Institute, Yerevan, Armenia}

S. Chatrchyan, V. Khachatryan, A.M. Sirunyan, A. Tumasyan

Institut für Hochenergiephysik der OeAW, Wien, Austria

W. Adam, E. Aguilo, T. Bergauer, M. Dragicevic, J. Erö, C. Fabjan ${ }^{1}$, M. Friedl, R. Frühwirth ${ }^{1}$, V.M. Ghete, J. Hammer, N. Hörmann, J. Hrubec, M. Jeitler ${ }^{1}$, W. Kiesenhofer, V. Knünz, M. Krammer ${ }^{1}$, I. Krätschmer, D. Liko, I. Mikulec, M. Pernicka ${ }^{\dagger}$, B. Rahbaran, C. Rohringer, H. Rohringer, R. Schöfbeck, J. Strauss, A. Taurok, W. Waltenberger, G. Walzel, E. Widl, C.-E. Wulz ${ }^{1}$

National Centre for Particle and High Energy Physics, Minsk, Belarus

V. Mossolov, N. Shumeiko, J. Suarez Gonzalez

\section{Universiteit Antwerpen, Antwerpen, Belgium}

M. Bansal, S. Bansal, T. Cornelis, E.A. De Wolf, X. Janssen, S. Luyckx, L. Mucibello, S. Ochesanu, B. Roland, R. Rougny, M. Selvaggi, Z. Staykova, H. Van Haevermaet, P. Van Mechelen, N. Van Remortel, A. Van Spilbeeck

\section{Vrije Universiteit Brussel, Brussel, Belgium}

F. Blekman, S. Blyweert, J. D'Hondt, R. Gonzalez Suarez, A. Kalogeropoulos, M. Maes, A. Olbrechts, W. Van Doninck, P. Van Mulders, G.P. Van Onsem, I. Villella

\section{Université Libre de Bruxelles, Bruxelles, Belgium}

B. Clerbaux, G. De Lentdecker, V. Dero, A.P.R. Gay, T. Hreus, A. Léonard, P.E. Marage, A. Mohammadi, T. Reis, L. Thomas, G. Vander Marcken, C. Vander Velde, P. Vanlaer, J. Wang

\section{Ghent University, Ghent, Belgium}

V. Adler, K. Beernaert, A. Cimmino, S. Costantini, G. Garcia, M. Grunewald, B. Klein, J. Lellouch, A. Marinov, J. Mccartin, A.A. Ocampo Rios, D. Ryckbosch, N. Strobbe, F. Thyssen, M. Tytgat, P. Verwilligen, S. Walsh, E. Yazgan, N. Zaganidis

\section{Université Catholique de Louvain, Louvain-la-Neuve, Belgium}

S. Basegmez, G. Bruno, R. Castello, L. Ceard, C. Delaere, T. du Pree, D. Favart, L. Forthomme, A. Giammanco ${ }^{2}$, J. Hollar, V. Lemaitre, J. Liao, O. Militaru, C. Nuttens, D. Pagano, A. Pin, K. Piotrzkowski, N. Schul, J.M. Vizan Garcia

\section{Université de Mons, Mons, Belgium}

N. Beliy, T. Caebergs, E. Daubie, G.H. Hammad

Centro Brasileiro de Pesquisas Fisicas, Rio de Janeiro, Brazil

G.A. Alves, M. Correa Martins Junior, D. De Jesus Damiao, T. Martins, M.E. Pol, M.H.G. Souza 
Universidade do Estado do Rio de Janeiro, Rio de Janeiro, Brazil

W.L. Aldá Júnior, W. Carvalho, A. Custódio, E.M. Da Costa, C. De Oliveira Martins, S. Fonseca De Souza, D. Matos Figueiredo, L. Mundim, H. Nogima, V. Oguri, W.L. Prado Da Silva, A. Santoro, L. Soares Jorge, A. Sznajder

Instituto de Fisica Teorica, Universidade Estadual Paulista, Sao Paulo, Brazil T.S. Anjos $^{3}$, C.A. Bernardes ${ }^{3}$, F.A. Dias ${ }^{4}$, T.R. Fernandez Perez Tomei, E.M. Gregores ${ }^{3}$, C. Lagana, F. Marinho, P.G. Mercadante ${ }^{3}$, S.F. Novaes, Sandra S. Padula

Institute for Nuclear Research and Nuclear Energy, Sofia, Bulgaria

V. Genchev ${ }^{5}$, P. Iaydjiev ${ }^{5}$, S. Piperov, M. Rodozov, S. Stoykova, G. Sultanov, V. Tcholakov,

R. Trayanov, M. Vutova

University of Sofia, Sofia, Bulgaria

A. Dimitrov, R. Hadjiiska, V. Kozhuharov, L. Litov, B. Pavlov, P. Petkov

Institute of High Energy Physics, Beijing, China

J.G. Bian, G.M. Chen, H.S. Chen, C.H. Jiang, D. Liang, S. Liang, X. Meng, J. Tao, J. Wang, X. Wang, Z. Wang, H. Xiao, M. Xu, J. Zang, Z. Zhang

State Key Lab. of Nucl. Phys. and Tech., Peking University, Beijing, China

C. Asawatangtrakuldee, Y. Ban, Y. Guo, W. Li, S. Liu, Y. Mao, S.J. Qian, H. Teng,

D. Wang, L. Zhang, W. Zou

Universidad de Los Andes, Bogota, Colombia

C. Avila, J.P. Gomez, B. Gomez Moreno, A.F. Osorio Oliveros, J.C. Sanabria

Technical University of Split, Split, Croatia

N. Godinovic, D. Lelas, R. Plestina ${ }^{6}$, D. Polic, I. Puljak ${ }^{5}$

University of Split, Split, Croatia

Z. Antunovic, M. Kovac

Institute Rudjer Boskovic, Zagreb, Croatia

V. Brigljevic, S. Duric, K. Kadija, J. Luetic, S. Morovic

University of Cyprus, Nicosia, Cyprus

A. Attikis, M. Galanti, G. Mavromanolakis, J. Mousa, C. Nicolaou, F. Ptochos, P.A. Razis

Charles University, Prague, Czech Republic

M. Finger, M. Finger Jr.

Academy of Scientific Research and Technology of the Arab Republic of Egypt, Egyptian Network of High Energy Physics, Cairo, Egypt

Y. Assran ${ }^{7}$, S. Elgammal ${ }^{8}$, A. Ellithi Kamel ${ }^{9}$, S. Khalil ${ }^{8}$, M.A. Mahmoud ${ }^{10}$, A. Radi ${ }^{11,12}$

National Institute of Chemical Physics and Biophysics, Tallinn, Estonia

M. Kadastik, M. Müntel, M. Raidal, L. Rebane, A. Tiko

Department of Physics, University of Helsinki, Helsinki, Finland

P. Eerola, G. Fedi, M. Voutilainen 


\section{Helsinki Institute of Physics, Helsinki, Finland}

J. Härkönen, A. Heikkinen, V. Karimäki, R. Kinnunen, M.J. Kortelainen, T. Lampén, K. Lassila-Perini, S. Lehti, T. Lindén, P. Luukka, T. Mäenpää, T. Peltola, E. Tuominen, J. Tuominiemi, E. Tuovinen, D. Ungaro, L. Wendland

\section{Lappeenranta University of Technology, Lappeenranta, Finland}

K. Banzuzi, A. Karjalainen, A. Korpela, T. Tuuva

DSM/IRFU, CEA/Saclay, Gif-sur-Yvette, France

M. Besancon, S. Choudhury, M. Dejardin, D. Denegri, B. Fabbro, J.L. Faure, F. Ferri, S. Ganjour, A. Givernaud, P. Gras, G. Hamel de Monchenault, P. Jarry, E. Locci, J. Malcles, L. Millischer, A. Nayak, J. Rander, A. Rosowsky, I. Shreyber, M. Titov

Laboratoire Leprince-Ringuet, Ecole Polytechnique, IN2P3-CNRS, Palaiseau, France

S. Baffioni, F. Beaudette, L. Benhabib, L. Bianchini, M. Bluj ${ }^{13}$, C. Broutin, P. Busson, C. Charlot, N. Daci, T. Dahms, L. Dobrzynski, R. Granier de Cassagnac, M. Haguenauer, P. Miné, C. Mironov, I.N. Naranjo, M. Nguyen, C. Ochando, P. Paganini, D. Sabes, R. Salerno, Y. Sirois, C. Veelken, A. Zabi

Institut Pluridisciplinaire Hubert Curien, Université de Strasbourg, Université de Haute Alsace Mulhouse, CNRS/IN2P3, Strasbourg, France

J.-L. Agram ${ }^{14}$, J. Andrea, D. Bloch, D. Bodin, J.-M. Brom, M. Cardaci, E.C. Chabert, C. Collard, E. Conte ${ }^{14}$, F. Drouhin ${ }^{14}$, C. Ferro, J.-C. Fontaine ${ }^{14}$, D. Gelé, U. Goerlach, P. Juillot, A.-C. Le Bihan, P. Van Hove

Centre de Calcul de l'Institut National de Physique Nucleaire et de Physique des Particules, CNRS/IN2P3, Villeurbanne, France, Villeurbanne, France

F. Fassi, D. Mercier

Université de Lyon, Université Claude Bernard Lyon 1, CNRS-IN2P3, Institut de Physique Nucléaire de Lyon, Villeurbanne, France

S. Beauceron, N. Beaupere, O. Bondu, G. Boudoul, J. Chasserat, R. Chierici ${ }^{5}$, D. Contardo, P. Depasse, H. El Mamouni, J. Fay, S. Gascon, M. Gouzevitch, B. Ille, T. Kurca, M. Lethuillier, L. Mirabito, S. Perries, L. Sgandurra, V. Sordini, Y. Tschudi, P. Verdier, S. Viret

Institute of High Energy Physics and Informatization, Tbilisi State University, Tbilisi, Georgia

Z. Tsamalaidze ${ }^{15}$

\section{RWTH Aachen University, I. Physikalisches Institut, Aachen, Germany}

G. Anagnostou, C. Autermann, S. Beranek, M. Edelhoff, L. Feld, N. Heracleous, O. Hindrichs, R. Jussen, K. Klein, J. Merz, A. Ostapchuk, A. Perieanu, F. Raupach, J. Sammet, S. Schael, D. Sprenger, H. Weber, B. Wittmer, V. Zhukov ${ }^{16}$ 
RWTH Aachen University, III. Physikalisches Institut A, Aachen, Germany M. Ata, J. Caudron, E. Dietz-Laursonn, D. Duchardt, M. Erdmann, R. Fischer, A. Güth, T. Hebbeker, C. Heidemann, K. Hoepfner, D. Klingebiel, P. Kreuzer, M. Merschmeyer, A. Meyer, M. Olschewski, P. Papacz, H. Pieta, H. Reithler, S.A. Schmitz, L. Sonnenschein, J. Steggemann, D. Teyssier, M. Weber

RWTH Aachen University, III. Physikalisches Institut B, Aachen, Germany M. Bontenackels, V. Cherepanov, Y. Erdogan, G. Flügge, H. Geenen, M. Geisler, W. Haj Ahmad, F. Hoehle, B. Kargoll, T. Kress, Y. Kuessel, J. Lingemann ${ }^{5}$, A. Nowack, L. Perchalla, O. Pooth, P. Sauerland, A. Stahl

\section{Deutsches Elektronen-Synchrotron, Hamburg, Germany}

M. Aldaya Martin, J. Behr, W. Behrenhoff, U. Behrens, M. Bergholz ${ }^{17}$, A. Bethani, K. Borras, A. Burgmeier, A. Cakir, L. Calligaris, A. Campbell, E. Castro, F. Costanza, D. Dammann, C. Diez Pardos, G. Eckerlin, D. Eckstein, G. Flucke, A. Geiser, I. Glushkov, P. Gunnellini, S. Habib, J. Hauk, G. Hellwig, H. Jung, M. Kasemann, P. Katsas, C. Kleinwort, H. Kluge, A. Knutsson, M. Krämer, D. Krücker, E. Kuznetsova, W. Lange, W. Lohmann ${ }^{17}$, B. Lutz, R. Mankel, I. Marfin, M. Marienfeld, I.-A. Melzer-Pellmann, A.B. Meyer, J. Mnich, A. Mussgiller, S. Naumann-Emme, O. Novgorodova, J. Olzem, H. Perrey, A. Petrukhin, D. Pitzl, A. Raspereza, P.M. Ribeiro Cipriano, C. Riedl, E. Ron, M. Rosin, J. Salfeld-Nebgen, R. Schmidt ${ }^{17}$, T. Schoerner-Sadenius, N. Sen, A. Spiridonov, M. Stein, R. Walsh, C. Wissing

\section{University of Hamburg, Hamburg, Germany}

V. Blobel, J. Draeger, H. Enderle, J. Erfle, U. Gebbert, M. Görner, T. Hermanns, R.S. Höing, K. Kaschube, G. Kaussen, H. Kirschenmann, R. Klanner, J. Lange, B. Mura, F. Nowak, T. Peiffer, N. Pietsch, D. Rathjens, C. Sander, H. Schettler, P. Schleper, E. Schlieckau, A. Schmidt, M. Schröder, T. Schum, M. Seidel, V. Sola, H. Stadie, G. Steinbrück, J. Thomsen, L. Vanelderen

\section{Institut für Experimentelle Kernphysik, Karlsruhe, Germany}

C. Barth, J. Berger, C. Böser, T. Chwalek, W. De Boer, A. Descroix, A. Dierlamm, M. Feindt, M. Guthoff ${ }^{5}$, C. Hackstein, F. Hartmann, T. Hauth ${ }^{5}$, M. Heinrich, H. Held, K.H. Hoffmann, U. Husemann, I. Katkov ${ }^{16}$, J.R. Komaragiri, P. Lobelle Pardo, D. Martschei, S. Mueller, Th. Müller, M. Niegel, A. Nürnberg, O. Oberst, A. Oehler, J. Ott, G. Quast, K. Rabbertz, F. Ratnikov, N. Ratnikova, S. Röcker, F.-P. Schilling, G. Schott, H.J. Simonis, F.M. Stober, D. Troendle, R. Ulrich, J. Wagner-Kuhr, S. Wayand, T. Weiler, M. Zeise

\section{Institute of Nuclear Physics "Demokritos", Aghia Paraskevi, Greece}

G. Daskalakis, T. Geralis, S. Kesisoglou, A. Kyriakis, D. Loukas, I. Manolakos, A. Markou, C. Markou, C. Mavrommatis, E. Ntomari

University of Athens, Athens, Greece

L. Gouskos, T.J. Mertzimekis, A. Panagiotou, N. Saoulidou 
University of Ioánnina, Ioánnina, Greece

I. Evangelou, C. Foudas, P. Kokkas, N. Manthos, I. Papadopoulos, V. Patras

KFKI Research Institute for Particle and Nuclear Physics, Budapest, Hungary G. Bencze, C. Hajdu, P. Hidas, D. Horvath ${ }^{18}$, F. Sikler, V. Veszpremi, G. Vesztergombi ${ }^{19}$

Institute of Nuclear Research ATOMKI, Debrecen, Hungary

N. Beni, S. Czellar, J. Molnar, J. Palinkas, Z. Szillasi

University of Debrecen, Debrecen, Hungary

J. Karancsi, P. Raics, Z.L. Trocsanyi, B. Ujvari

Panjab University, Chandigarh, India

S.B. Beri, V. Bhatnagar, N. Dhingra, R. Gupta, M. Kaur, M.Z. Mehta, N. Nishu, L.K. Saini, A. Sharma, J.B. Singh

University of Delhi, Delhi, India

Ashok Kumar, Arun Kumar, S. Ahuja, A. Bhardwaj, B.C. Choudhary, S. Malhotra, M. Naimuddin, K. Ranjan, V. Sharma, R.K. Shivpuri

Saha Institute of Nuclear Physics, Kolkata, India

S. Banerjee, S. Bhattacharya, S. Dutta, B. Gomber, Sa. Jain, Sh. Jain, R. Khurana, S. Sarkar, M. Sharan

Bhabha Atomic Research Centre, Mumbai, India

A. Abdulsalam, R.K. Choudhury, D. Dutta, S. Kailas, V. Kumar, P. Mehta, A.K. Mohanty ${ }^{5}$, L.M. Pant, P. Shukla

Tata Institute of Fundamental Research - EHEP, Mumbai, India

T. Aziz, S. Ganguly, M. Guchait ${ }^{20}$, M. Maity ${ }^{21}$, G. Majumder, K. Mazumdar, G.B. Mohanty, B. Parida, K. Sudhakar, N. Wickramage

Tata Institute of Fundamental Research - HECR, Mumbai, India

S. Banerjee, S. Dugad

Institute for Research in Fundamental Sciences (IPM), Tehran, Iran

H. Arfaei ${ }^{22}$, H. Bakhshiansohi, S.M. Etesami ${ }^{23}$, A. Fahim ${ }^{22}$, M. Hashemi, H. Hesari, A. Jafari, M. Khakzad, M. Mohammadi Najafabadi, S. Paktinat Mehdiabadi, B. Safarzadeh ${ }^{24}$, M. Zeinali

INFN Sezione di Bari ${ }^{a}$, Università di Bari ${ }^{b}$, Politecnico di Bari ${ }^{c}$, Bari, Italy M. Abbrescia ${ }^{a, b}$, L. Barbone ${ }^{a, b}$, C. Calabria ${ }^{a, b, 5}$, S.S. Chhibra ${ }^{a, b}$, A. Colaleo ${ }^{a}$, D. Creanza ${ }^{a, c}$, N. De Filippis ${ }^{a, c, 5}$, M. De Palma ${ }^{a, b}$, L. Fiore $^{a}$, G. Iaselli ${ }^{a, c}$, L. Lusito $^{a, b}$, G. Maggi ${ }^{a, c}$, M. Maggi ${ }^{a}$, B. Marangelli ${ }^{a, b}$, S. $\mathrm{My}^{a, c}$, S. Nuzzo ${ }^{a, b}$, N. Pacifico ${ }^{a, b}$, A. Pompili ${ }^{a, b}$, G. Pugliese ${ }^{a, c}$, G. Selvaggi ${ }^{a, b}$, L. Silvestris ${ }^{a}$, G. $\operatorname{Singh}^{a, b}$, R. Venditti ${ }^{a, b}$, G. Zito ${ }^{a}$

INFN Sezione di Bologna ${ }^{a}$, Università di Bologna ${ }^{b}$, Bologna, Italy

G. Abbiendi ${ }^{a}$, A.C. Benvenuti ${ }^{a}$, D. Bonacorsi ${ }^{a, b}$, S. Braibant-Giacomelli ${ }^{a, b}$, L. Brigliadori ${ }^{a, b}$, P. Capiluppi ${ }^{a, b}$, A. Castro ${ }^{a, b}$, F.R. Cavallo ${ }^{a}$, M. Cuffiani ${ }^{a, b}$, 
G.M. Dallavalle ${ }^{a}$, F. Fabbri ${ }^{a}$, A. Fanfani ${ }^{a, b}$, D. Fasanella ${ }^{a, b, 5}$, P. Giacomelli ${ }^{a}$, C. Grandi ${ }^{a}$, L. Guiducci ${ }^{a}, b$, S. Marcellini ${ }^{a}$, G. Masetti ${ }^{a}$, M. Meneghelli ${ }^{a, b, 5}$, A. Montanari $^{a}$,

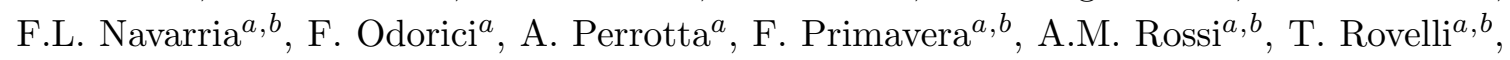
G.P. Siroli ${ }^{a, b}$, R. Travaglini ${ }^{a, b}$

INFN Sezione di Catania ${ }^{a}$, Università di Catania ${ }^{b}$, Catania, Italy

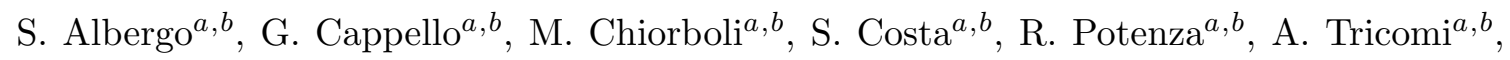
C. Tuve ${ }^{a, b}$

INFN Sezione di Firenze ${ }^{a}$, Università di Firenze ${ }^{b}$, Firenze, Italy

G. Barbagli ${ }^{a}$, V. Ciulli ${ }^{a, b}$, C. Civinini ${ }^{a}$, R. D’Alessandro ${ }^{a, b}$, E. Focardi ${ }^{a, b}$, S. Frosali ${ }^{a, b}$, E. Gallo ${ }^{a}$, S. Gonzi ${ }^{a, b}$, M. Meschini ${ }^{a}$, S. Paoletti ${ }^{a}$, G. Sguazzoni ${ }^{a}$, A. Tropiano $^{a, b}$

INFN Laboratori Nazionali di Frascati, Frascati, Italy

L. Benussi, S. Bianco, S. Colafranceschi ${ }^{25}$, F. Fabbri, D. Piccolo

INFN Sezione di Genova ${ }^{a}$, Università di Genova ${ }^{b}$, Genova, Italy

P. Fabbricatore ${ }^{a}$, R. Musenich ${ }^{a}$, S. Tosi ${ }^{a, b}$

INFN Sezione di Milano-Bicocca ${ }^{a}$, Università di Milano-Bicocca ${ }^{b}$, Milano, Italy

A. Benaglia ${ }^{a, b}$, F. De Guio ${ }^{a, b}$, L. Di Matteo ${ }^{a, b, 5}$, S. Fiorendi ${ }^{a, b}$, S. Gennai ${ }^{a, 5}$, A. Ghezzi $^{a, b}$, S. Malvezzi ${ }^{a}$, R.A. Manzoni ${ }^{a, b}$, A. Martelli ${ }^{a}, b$, A. Massironi ${ }^{a, b, 5}$, D. Menasce $^{a}$, L. Moroni ${ }^{a}$, M. Paganoni ${ }^{a, b}$, D. Pedrini ${ }^{a}$, S. Ragazzi $^{a, b}$, N. Redaelli ${ }^{a}$, S. Sala ${ }^{a}$, T. Tabarelli de Fatis $^{a, b}$

INFN Sezione di Napoli ${ }^{a}$, Università di Napoli "Federico II" ${ }^{b}$, Napoli, Italy S. Buontempo ${ }^{a}$, C.A. Carrillo Montoya ${ }^{a}$, N. Cavallo ${ }^{a, 26}$, A. De Cosa $^{a, b, 5}$, O. Dogangun ${ }^{a, b}$,

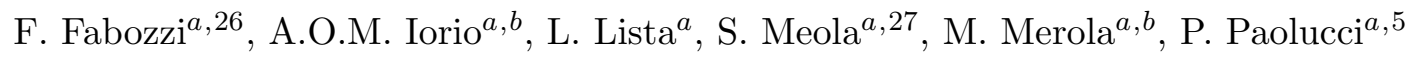

INFN Sezione di Padova ${ }^{a}$, Università di Padova ${ }^{b}$, Università di Trento (Trento) ${ }^{c}$, Padova, Italy

P. Azzi ${ }^{a}$, N. Bacchetta ${ }^{a, 5}$, P. Bellan ${ }^{a, b}$, D. Bisello ${ }^{a, b}$, A. Branca ${ }^{a, b, 5}$, R. Carlin $^{a, b}$,

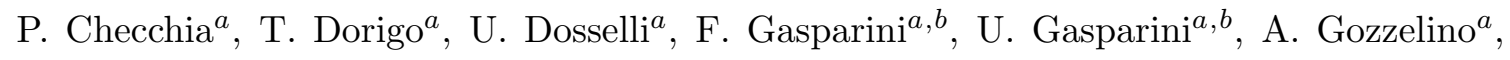
K. Kanishchev ${ }^{a, c}$, S. Lacaprara ${ }^{a}$, I. Lazzizzera ${ }^{a, c}$, M. Margoni $^{a, b}$, A.T. Meneguzzo ${ }^{a, b}$, M. Nespolo ${ }^{a, 5}$, J. Pazzini ${ }^{a, b}$, P. Ronchese ${ }^{a, b}$, F. Simonetto ${ }^{a, b}$, E. Torassa ${ }^{a}$, S. Vanini ${ }^{a, b}$, P. Zotto ${ }^{a, b}$, G. Zumerle ${ }^{a, b}$

INFN Sezione di Pavia ${ }^{a}$, Università di Pavia ${ }^{b}$, Pavia, Italy

M. Gabusi ${ }^{a}, b$, S.P. Ratti ${ }^{a, b}$, C. Riccardi ${ }^{a, b}$, P. Torre ${ }^{a, b}$, P. Vitulo ${ }^{a, b}$

INFN Sezione di Perugia ${ }^{a}$, Università di Perugia ${ }^{b}$, Perugia, Italy

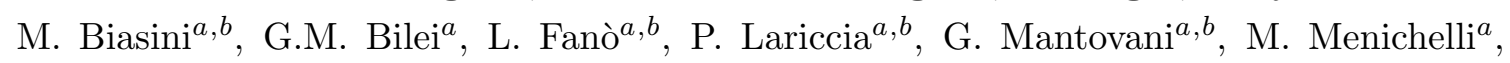
A. Nappi ${ }^{a, b \dagger}$, F. Romeo ${ }^{a, b}$, A. Saha ${ }^{a}$, A. Santocchia ${ }^{a, b}$, A. Spiezia ${ }^{a, b}$, S. Taroni ${ }^{a, b}$

INFN Sezione di Pisa ${ }^{a}$, Università di Pisa ${ }^{b}$, Scuola Normale Superiore di Pisa ${ }^{c}$, Pisa, Italy

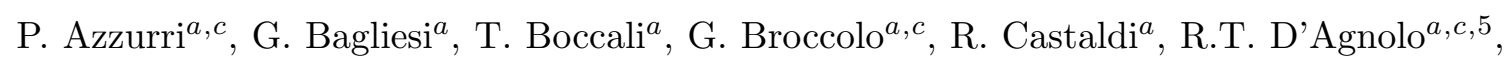

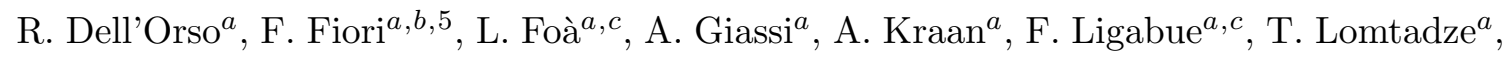




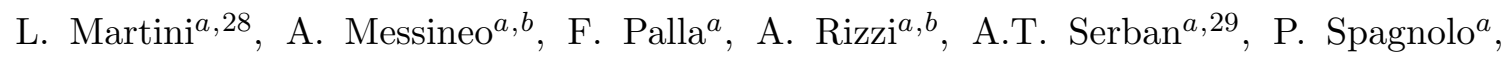

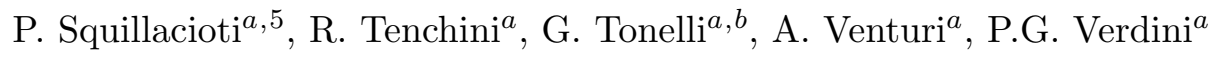

INFN Sezione di Roma ${ }^{a}$, Università di Roma ${ }^{b}$, Roma, Italy

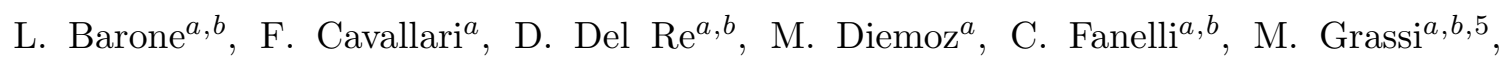
E. Longo $^{a, b}$, P. Meridiani ${ }^{a, 5}$, F. Micheli ${ }^{a, b}$, S. Nourbakhsh ${ }^{a, b}$, G. Organtini ${ }^{a, b}$,

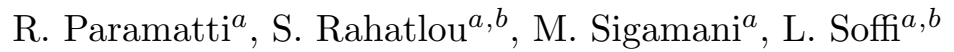

INFN Sezione di Torino ${ }^{a}$, Università di Torino ${ }^{b}$, Università del Piemonte Orientale (Novara) ${ }^{c}$, Torino, Italy

N. Amapane ${ }^{a, b}$, R. Arcidiacono ${ }^{a, c}, \mathrm{~S}$. Argiro ${ }^{a, b}$, M. Arneodo $^{a, c}$, C. Biino $^{a}$, N. Cartiglia ${ }^{a}$, M. Costa ${ }^{a, b}$, N. Demaria ${ }^{a}$, C. Mariotti ${ }^{a, 5}$, S. Maselli ${ }^{a}$, E. Migliore ${ }^{a, b}$, V. Monaco ${ }^{a, b}$,

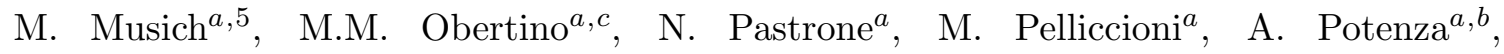
A. Romero ${ }^{a, b}$, M. Ruspa ${ }^{a, c}$, R. Sacchi ${ }^{a, b}$, A. Solano ${ }^{a, b}$, A. Staiano ${ }^{a}$, A. Vilela Pereira ${ }^{a}$

INFN Sezione di Trieste ${ }^{a}$, Università di Trieste ${ }^{b}$, Trieste, Italy

S. Belforte ${ }^{a}$, V. Candelise ${ }^{a, b}$, M. Casarsa ${ }^{a}$, F. Cossutti ${ }^{a}$, G. Della Ricca ${ }^{a, b}$, B. Gobbo ${ }^{a}$, M. Marone ${ }^{a, b, 5}$, D. Montanino ${ }^{a, b, 5}$, A. Penzo $^{a}$, A. Schizzi ${ }^{a, b}$

Kangwon National University, Chunchon, Korea

S.G. Heo, T.Y. Kim, S.K. Nam

Kyungpook National University, Daegu, Korea

S. Chang, D.H. Kim, G.N. Kim, D.J. Kong, H. Park, S.R. Ro, D.C. Son, T. Son

Chonnam National University, Institute for Universe and Elementary Particles, Kwangju, Korea

J.Y. Kim, Zero J. Kim, S. Song

Korea University, Seoul, Korea

S. Choi, D. Gyun, B. Hong, M. Jo, H. Kim, T.J. Kim, K.S. Lee, D.H. Moon, S.K. Park

University of Seoul, Seoul, Korea

M. Choi, J.H. Kim, C. Park, I.C. Park, S. Park, G. Ryu

Sungkyunkwan University, Suwon, Korea

Y. Cho, Y. Choi, Y.K. Choi, J. Goh, M.S. Kim, E. Kwon, B. Lee, J. Lee, S. Lee, H. Seo, I. $\mathrm{Yu}$

Vilnius University, Vilnius, Lithuania

M.J. Bilinskas, I. Grigelionis, M. Janulis, A. Juodagalvis

Centro de Investigacion y de Estudios Avanzados del IPN, Mexico City, Mexico

H. Castilla-Valdez, E. De La Cruz-Burelo, I. Heredia-de La Cruz, R. Lopez-Fernandez,

R. Magaña Villalba, J. Martínez-Ortega, A. Sánchez-Hernández, L.M. Villasenor-Cendejas

Universidad Iberoamericana, Mexico City, Mexico

S. Carrillo Moreno, F. Vazquez Valencia

Benemerita Universidad Autonoma de Puebla, Puebla, Mexico

H.A. Salazar Ibarguen 
Universidad Autónoma de San Luis Potosí, San Luis Potosí, Mexico

E. Casimiro Linares, A. Morelos Pineda, M.A. Reyes-Santos

University of Auckland, Auckland, New Zealand

D. Krofcheck

University of Canterbury, Christchurch, New Zealand

A.J. Bell, P.H. Butler, R. Doesburg, S. Reucroft, H. Silverwood

National Centre for Physics, Quaid-I-Azam University, Islamabad, Pakistan

M. Ahmad, M.H. Ansari, M.I. Asghar, H.R. Hoorani, S. Khalid, W.A. Khan, T. Khurshid, S. Qazi, M.A. Shah, M. Shoaib

National Centre for Nuclear Research, Swierk, Poland

H. Bialkowska, B. Boimska, T. Frueboes, R. Gokieli, M. Górski, M. Kazana, K. Nawrocki,

K. Romanowska-Rybinska, M. Szleper, G. Wrochna, P. Zalewski

Institute of Experimental Physics, Faculty of Physics, University of Warsaw, Warsaw, Poland

G. Brona, K. Bunkowski, M. Cwiok, W. Dominik, K. Doroba, A. Kalinowski, M. Konecki, J. Krolikowski

Laboratório de Instrumentação e Física Experimental de Partículas, Lisboa, Portugal

N. Almeida, P. Bargassa, A. David, P. Faccioli, P.G. Ferreira Parracho, M. Gallinaro, J. Seixas, J. Varela, P. Vischia

Joint Institute for Nuclear Research, Dubna, Russia

I. Belotelov, P. Bunin, M. Gavrilenko, I. Golutvin, I. Gorbunov, A. Kamenev, V. Karjavin, G. Kozlov, A. Lanev, A. Malakhov, P. Moisenz, V. Palichik, V. Perelygin, S. Shmatov, V. Smirnov, A. Volodko, A. Zarubin

Petersburg Nuclear Physics Institute, Gatchina (St. Petersburg), Russia

S. Evstyukhin, V. Golovtsov, Y. Ivanov, V. Kim, P. Levchenko, V. Murzin, V. Oreshkin, I. Smirnov, V. Sulimov, L. Uvarov, S. Vavilov, A. Vorobyev, An. Vorobyev

Institute for Nuclear Research, Moscow, Russia

Yu. Andreev, A. Dermenev, S. Gninenko, N. Golubev, M. Kirsanov, N. Krasnikov, V. Matveev, A. Pashenkov, D. Tlisov, A. Toropin

Institute for Theoretical and Experimental Physics, Moscow, Russia

V. Epshteyn, M. Erofeeva, V. Gavrilov, M. Kossov, N. Lychkovskaya, V. Popov, G. Safronov, S. Semenov, V. Stolin, E. Vlasov, A. Zhokin

Moscow State University, Moscow, Russia

A. Belyaev, E. Boos, M. Dubinin ${ }^{4}$, L. Dudko, A. Ershov, A. Gribushin, V. Klyukhin, O. Kodolova, I. Lokhtin, A. Markina, S. Obraztsov, M. Perfilov, S. Petrushanko, A. Popov, L. Sarycheva ${ }^{\dagger}$, V. Savrin, A. Snigirev 
P.N. Lebedev Physical Institute, Moscow, Russia

V. Andreev, M. Azarkin, I. Dremin, M. Kirakosyan, A. Leonidov, G. Mesyats, S.V. Rusakov, A. Vinogradov

State Research Center of Russian Federation, Institute for High Energy Physics, Protvino, Russia

I. Azhgirey, I. Bayshev, S. Bitioukov, V. Grishin ${ }^{5}$, V. Kachanov, D. Konstantinov,

V. Krychkine, V. Petrov, R. Ryutin, A. Sobol, L. Tourtchanovitch, S. Troshin, N. Tyurin,

A. Uzunian, A. Volkov

University of Belgrade, Faculty of Physics and Vinca Institute of Nuclear Sciences, Belgrade, Serbia

P. Adzic ${ }^{30}$, M. Djordjevic, M. Ekmedzic, D. Krpic ${ }^{30}$, J. Milosevic

Centro de Investigaciones Energéticas Medioambientales y Tecnológicas (CIEMAT), Madrid, Spain

M. Aguilar-Benitez, J. Alcaraz Maestre, P. Arce, C. Battilana, E. Calvo, M. Cerrada,

M. Chamizo Llatas, N. Colino, B. De La Cruz, A. Delgado Peris, D. Domínguez Vázquez,

C. Fernandez Bedoya, J.P. Fernández Ramos, A. Ferrando, J. Flix, M.C. Fouz, P. GarciaAbia, O. Gonzalez Lopez, S. Goy Lopez, J.M. Hernandez, M.I. Josa, G. Merino, J. Puerta Pelayo, A. Quintario Olmeda, I. Redondo, L. Romero, J. Santaolalla, M.S. Soares, C. Willmott

Universidad Autónoma de Madrid, Madrid, Spain

C. Albajar, G. Codispoti, J.F. de Trocóniz

Universidad de Oviedo, Oviedo, Spain

H. Brun, J. Cuevas, J. Fernandez Menendez, S. Folgueras, I. Gonzalez Caballero, L. Lloret Iglesias, J. Piedra Gomez

Instituto de Física de Cantabria (IFCA), CSIC-Universidad de Cantabria, Santander, Spain

J.A. Brochero Cifuentes, I.J. Cabrillo, A. Calderon, S.H. Chuang, J. Duarte Campderros, M. Felcini ${ }^{31}$, M. Fernandez, G. Gomez, J. Gonzalez Sanchez, A. Graziano, C. Jorda, A. Lopez Virto, J. Marco, R. Marco, C. Martinez Rivero, F. Matorras, F.J. Munoz Sanchez, T. Rodrigo, A.Y. Rodríguez-Marrero, A. Ruiz-Jimeno, L. Scodellaro, I. Vila, R. Vilar Cortabitarte

\section{CERN, European Organization for Nuclear Research, Geneva, Switzerland}

D. Abbaneo, E. Auffray, G. Auzinger, M. Bachtis, P. Baillon, A.H. Ball, D. Barney, J.F. Benitez, C. Bernet ${ }^{6}$, G. Bianchi, P. Bloch, A. Bocci, A. Bonato, C. Botta, H. Breuker, T. Camporesi, G. Cerminara, T. Christiansen, J.A. Coarasa Perez, D. D'Enterria, A. Dabrowski, A. De Roeck, S. Di Guida, M. Dobson, N. Dupont-Sagorin, A. ElliottPeisert, B. Frisch, W. Funk, G. Georgiou, M. Giffels, D. Gigi, K. Gill, D. Giordano, M. Girone, M. Giunta, F. Glege, R. Gomez-Reino Garrido, P. Govoni, S. Gowdy, R. Guida, M. Hansen, P. Harris, C. Hartl, J. Harvey, B. Hegner, A. Hinzmann, V. Innocente, P. Janot, K. Kaadze, E. Karavakis, K. Kousouris, P. Lecoq, Y.-J. Lee, P. Lenzi, C. Lourenço, 
N. Magini, T. Mäki, M. Malberti, L. Malgeri, M. Mannelli, L. Masetti, F. Meijers, S. Mersi, E. Meschi, R. Moser, M.U. Mozer, M. Mulders, P. Musella, E. Nesvold, T. Orimoto, L. Orsini, E. Palencia Cortezon, E. Perez, L. Perrozzi, A. Petrilli, A. Pfeiffer, M. Pierini, M. Pimiä, D. Piparo, G. Polese, L. Quertenmont, A. Racz, W. Reece, J. Rodrigues Antunes, G. Rolandi ${ }^{32}$, C. Rovelli ${ }^{33}$, M. Rovere, H. Sakulin, F. Santanastasio, C. Schäfer, C. Schwick, I. Segoni, S. Sekmen, A. Sharma, P. Siegrist, P. Silva, M. Simon, P. Sphicas ${ }^{34}$, D. Spiga, A. Tsirou, G.I. Veres ${ }^{19}$, J.R. Vlimant, H.K. Wöhri, S.D. Worm ${ }^{35}$, W.D. Zeuner

\section{Paul Scherrer Institut, Villigen, Switzerland}

W. Bertl, K. Deiters, W. Erdmann, K. Gabathuler, R. Horisberger, Q. Ingram, H.C. Kaestli, S. König, D. Kotlinski, U. Langenegger, F. Meier, D. Renker, T. Rohe, J. Sibille ${ }^{36}$

\section{Institute for Particle Physics, ETH Zurich, Zurich, Switzerland}

L. Bäni, P. Bortignon, M.A. Buchmann, B. Casal, N. Chanon, A. Deisher, G. Dissertori, M. Dittmar, M. Donegà, M. Dünser, J. Eugster, K. Freudenreich, C. Grab, D. Hits, P. Lecomte, W. Lustermann, A.C. Marini, P. Martinez Ruiz del Arbol, N. Mohr, F. Moortgat, C. Nägeli ${ }^{37}$, P. Nef, F. Nessi-Tedaldi, F. Pandolfi, L. Pape, F. Pauss, M. Peruzzi, F.J. Ronga, M. Rossini, L. Sala, A.K. Sanchez, A. Starodumov ${ }^{38}$, B. Stieger, M. Takahashi, L. Tauscher ${ }^{\dagger}$, A. Thea, K. Theofilatos, D. Treille, C. Urscheler, R. Wallny, H.A. Weber, L. Wehrli

\section{Universität Zürich, Zurich, Switzerland}

C. Amsler, V. Chiochia, S. De Visscher, C. Favaro, M. Ivova Rikova, B. Millan Mejias, P. Otiougova, P. Robmann, H. Snoek, S. Tupputi, M. Verzetti

\section{National Central University, Chung-Li, Taiwan}

Y.H. Chang, K.H. Chen, C.M. Kuo, S.W. Li, W. Lin, Z.K. Liu, Y.J. Lu, D. Mekterovic, A.P. Singh, R. Volpe, S.S. Yu

\section{National Taiwan University (NTU), Taipei, Taiwan}

P. Bartalini, P. Chang, Y.H. Chang, Y.W. Chang, Y. Chao, K.F. Chen, C. Dietz, U. Grundler, W.-S. Hou, Y. Hsiung, K.Y. Kao, Y.J. Lei, R.-S. Lu, D. Majumder, E. Petrakou, X. Shi, J.G. Shiu, Y.M. Tzeng, X. Wan, M. Wang

\section{Chulalongkorn University, Bangkok, Thailand}

B. Asavapibhop, N. Srimanobhas

\section{Cukurova University, Adana, Turkey}

A. Adiguzel, M.N. Bakirci ${ }^{39}$, S. Cerci ${ }^{40}$, C. Dozen, I. Dumanoglu, E. Eskut, S. Girgis, G. Gokbulut, E. Gurpinar, I. Hos, E.E. Kangal, T. Karaman, G. Karapinar ${ }^{41}$, A. Kayis Topaksu, G. Onengut, K. Ozdemir, S. Ozturk ${ }^{42}$, A. Polatoz, K. Sogut ${ }^{43}$, D. Sunar Cerci ${ }^{40}$, B. Tali ${ }^{40}$, H. Topakli ${ }^{39}$, L.N. Vergili, M. Vergili

\section{Middle East Technical University, Physics Department, Ankara, Turkey}

I.V. Akin, T. Aliev, B. Bilin, S. Bilmis, M. Deniz, H. Gamsizkan, A.M. Guler, K. Ocalan, A. Ozpineci, M. Serin, R. Sever, U.E. Surat, M. Yalvac, E. Yildirim, M. Zeyrek 
Bogazici University, Istanbul, Turkey

E. Gülmez, B. Isildak ${ }^{44}$, M. Kaya ${ }^{45}$, O. Kaya ${ }^{45}$, S. Ozkorucuklu ${ }^{46}$, N. Sonmez ${ }^{47}$

Istanbul Technical University, Istanbul, Turkey

K. Cankocak

National Scientific Center, Kharkov Institute of Physics and Technology, Kharkov, Ukraine

L. Levchuk

\section{University of Bristol, Bristol, United Kingdom}

F. Bostock, J.J. Brooke, E. Clement, D. Cussans, H. Flacher, R. Frazier, J. Goldstein, M. Grimes, G.P. Heath, H.F. Heath, L. Kreczko, S. Metson, D.M. Newbold ${ }^{35}$, K. Nirunpong, A. Poll, S. Senkin, V.J. Smith, T. Williams

\section{Rutherford Appleton Laboratory, Didcot, United Kingdom}

L. Basso ${ }^{48}$, K.W. Bell, A. Belyaev ${ }^{48}$, C. Brew, R.M. Brown, D.J.A. Cockerill, J.A. Coughlan, K. Harder, S. Harper, J. Jackson, B.W. Kennedy, E. Olaiya, D. Petyt, B.C. RadburnSmith, C.H. Shepherd-Themistocleous, I.R. Tomalin, W.J. Womersley

\section{Imperial College, London, United Kingdom}

R. Bainbridge, G. Ball, R. Beuselinck, O. Buchmuller, D. Colling, N. Cripps, M. Cutajar, P. Dauncey, G. Davies, M. Della Negra, W. Ferguson, J. Fulcher, D. Futyan, A. Gilbert, A. Guneratne Bryer, G. Hall, Z. Hatherell, J. Hays, G. Iles, M. Jarvis, G. Karapostoli, L. Lyons, A.-M. Magnan, J. Marrouche, B. Mathias, R. Nandi, J. Nash, A. Nikitenko ${ }^{38}$, A. Papageorgiou, J. Pela, M. Pesaresi, K. Petridis, M. Pioppi ${ }^{49}$, D.M. Raymond, S. Rogerson, A. Rose, M.J. Ryan, C. Seez, P. Sharp ${ }^{\dagger}$, A. Sparrow, M. Stoye, A. Tapper, M. Vazquez Acosta, T. Virdee, S. Wakefield, N. Wardle, T. Whyntie

\section{Brunel University, Uxbridge, United Kingdom}

M. Chadwick, J.E. Cole, P.R. Hobson, A. Khan, P. Kyberd, D. Leggat, D. Leslie, W. Martin, I.D. Reid, P. Symonds, L. Teodorescu, M. Turner

Baylor University, Waco, USA

K. Hatakeyama, H. Liu, T. Scarborough

The University of Alabama, Tuscaloosa, USA

O. Charaf, C. Henderson, P. Rumerio

\section{Boston University, Boston, USA}

A. Avetisyan, T. Bose, C. Fantasia, A. Heister, J. St. John, P. Lawson, D. Lazic, J. Rohlf, D. Sperka, L. Sulak

\section{Brown University, Providence, USA}

J. Alimena, S. Bhattacharya, D. Cutts, Z. Demiragli, A. Ferapontov, U. Heintz, S. Jabeen, G. Kukartsev, E. Laird, G. Landsberg, M. Luk, M. Narain, D. Nguyen, M. Segala, T. Sinthuprasith, T. Speer, K.V. Tsang 


\section{University of California, Davis, Davis, USA}

R. Breedon, G. Breto, M. Calderon De La Barca Sanchez, S. Chauhan, M. Chertok, J. Conway, R. Conway, P.T. Cox, J. Dolen, R. Erbacher, M. Gardner, R. Houtz, W. Ko, A. Kopecky, R. Lander, O. Mall, T. Miceli, D. Pellett, F. Ricci-tam, B. Rutherford, M. Searle, J. Smith, M. Squires, M. Tripathi, R. Vasquez Sierra, R. Yohay

University of California, Los Angeles, Los Angeles, USA

V. Andreev, D. Cline, R. Cousins, J. Duris, S. Erhan, P. Everaerts, C. Farrell, J. Hauser, M. Ignatenko, C. Jarvis, C. Plager, G. Rakness, P. Schlein ${ }^{\dagger}$, P. Traczyk, V. Valuev, M. Weber

\section{University of California, Riverside, Riverside, USA}

J. Babb, R. Clare, M.E. Dinardo, J. Ellison, J.W. Gary, F. Giordano, G. Hanson, G.Y. Jeng ${ }^{50}$, H. Liu, O.R. Long, A. Luthra, H. Nguyen, S. Paramesvaran, J. Sturdy, S. Sumowidagdo, R. Wilken, S. Wimpenny

\section{University of California, San Diego, La Jolla, USA}

W. Andrews, J.G. Branson, G.B. Cerati, S. Cittolin, D. Evans, F. Golf, A. Holzner, R. Kelley, M. Lebourgeois, J. Letts, I. Macneill, B. Mangano, S. Padhi, C. Palmer, G. Petrucciani, M. Pieri, M. Sani, V. Sharma, S. Simon, E. Sudano, M. Tadel, Y. Tu, A. Vartak, S. Wasserbaech ${ }^{51}$, F. Würthwein, A. Yagil, J. Yoo

\section{University of California, Santa Barbara, Santa Barbara, USA}

D. Barge, R. Bellan, C. Campagnari, M. D’Alfonso, T. Danielson, K. Flowers, P. Geffert, J. Incandela, C. Justus, P. Kalavase, S.A. Koay, D. Kovalskyi, V. Krutelyov, S. Lowette, N. Mccoll, V. Pavlunin, F. Rebassoo, J. Ribnik, J. Richman, R. Rossin, D. Stuart, W. To, C. West

\section{California Institute of Technology, Pasadena, USA}

A. Apresyan, A. Bornheim, Y. Chen, E. Di Marco, J. Duarte, M. Gataullin, Y. Ma, A. Mott, H.B. Newman, C. Rogan, M. Spiropulu, V. Timciuc, J. Veverka, R. Wilkinson, S. Xie, Y. Yang, R.Y. Zhu

\section{Carnegie Mellon University, Pittsburgh, USA}

B. Akgun, V. Azzolini, A. Calamba, R. Carroll, T. Ferguson, Y. Iiyama, D.W. Jang, Y.F. Liu, M. Paulini, H. Vogel, I. Vorobiev

\section{University of Colorado at Boulder, Boulder, USA}

J.P. Cumalat, B.R. Drell, W.T. Ford, A. Gaz, E. Luiggi Lopez, J.G. Smith, K. Stenson, K.A. Ulmer, S.R. Wagner

\section{Cornell University, Ithaca, USA}

J. Alexander, A. Chatterjee, N. Eggert, L.K. Gibbons, B. Heltsley, A. Khukhunaishvili, B. Kreis, N. Mirman, G. Nicolas Kaufman, J.R. Patterson, A. Ryd, E. Salvati, W. Sun, W.D. Teo, J. Thom, J. Thompson, J. Tucker, J. Vaughan, Y. Weng, L. Winstrom, P. Wittich 


\section{Fairfield University, Fairfield, USA}

D. Winn

\section{Fermi National Accelerator Laboratory, Batavia, USA}

S. Abdullin, M. Albrow, J. Anderson, L.A.T. Bauerdick, A. Beretvas, J. Berryhill, P.C. Bhat, I. Bloch, K. Burkett, J.N. Butler, V. Chetluru, H.W.K. Cheung, F. Chlebana, V.D. Elvira, I. Fisk, J. Freeman, Y. Gao, D. Green, O. Gutsche, J. Hanlon, R.M. Harris, J. Hirschauer, B. Hooberman, S. Jindariani, M. Johnson, U. Joshi, B. Kilminster, B. Klima, S. Kunori, S. Kwan, C. Leonidopoulos, J. Linacre, D. Lincoln, R. Lipton, J. Lykken, K. Maeshima, J.M. Marraffino, S. Maruyama, D. Mason, P. McBride, K. Mishra, S. Mrenna, Y. Musienko ${ }^{52}$, C. Newman-Holmes, V. O'Dell, O. Prokofyev, E. SextonKennedy, S. Sharma, W.J. Spalding, L. Spiegel, L. Taylor, S. Tkaczyk, N.V. Tran, L. Uplegger, E.W. Vaandering, R. Vidal, J. Whitmore, W. Wu, F. Yang, F. Yumiceva, J.C. Yun

\section{University of Florida, Gainesville, USA}

D. Acosta, P. Avery, D. Bourilkov, M. Chen, T. Cheng, S. Das, M. De Gruttola, G.P. Di Giovanni, D. Dobur, A. Drozdetskiy, R.D. Field, M. Fisher, Y. Fu, I.K. Furic, J. Gartner, J. Hugon, B. Kim, J. Konigsberg, A. Korytov, A. Kropivnitskaya, T. Kypreos, J.F. Low, K. Matchev, P. Milenovic ${ }^{53}$, G. Mitselmakher, L. Muniz, M. Park, R. Remington, A. Rinkevicius, P. Sellers, N. Skhirtladze, M. Snowball, J. Yelton, M. Zakaria

Florida International University, Miami, USA

V. Gaultney, S. Hewamanage, L.M. Lebolo, S. Linn, P. Markowitz, G. Martinez, J.L. Rodriguez

\section{Florida State University, Tallahassee, USA}

T. Adams, A. Askew, J. Bochenek, J. Chen, B. Diamond, S.V. Gleyzer, J. Haas, S. Hagopian, V. Hagopian, M. Jenkins, K.F. Johnson, H. Prosper, V. Veeraraghavan, M. Weinberg

\section{Florida Institute of Technology, Melbourne, USA}

M.M. Baarmand, B. Dorney, M. Hohlmann, H. Kalakhety, I. Vodopiyanov

\section{University of Illinois at Chicago (UIC), Chicago, USA}

M.R. Adams, I.M. Anghel, L. Apanasevich, Y. Bai, V.E. Bazterra, R.R. Betts, I. Bucinskaite, J. Callner, R. Cavanaugh, O. Evdokimov, L. Gauthier, C.E. Gerber, D.J. Hofman, S. Khalatyan, F. Lacroix, M. Malek, C. O'Brien, C. Silkworth, D. Strom, P. Turner, N. Varelas

\section{The University of Iowa, Iowa City, USA}

U. Akgun, E.A. Albayrak, B. Bilki ${ }^{54}$, W. Clarida, F. Duru, J.-P. Merlo, H. Mermerkaya ${ }^{55}$, A. Mestvirishvili, A. Moeller, J. Nachtman, C.R. Newsom, E. Norbeck, Y. Onel, F. Ozok ${ }^{56}$, S. Sen, P. Tan, E. Tiras, J. Wetzel, T. Yetkin, K. Yi

\section{Johns Hopkins University, Baltimore, USA}

B.A. Barnett, B. Blumenfeld, S. Bolognesi, D. Fehling, G. Giurgiu, A.V. Gritsan, Z.J. Guo, G. Hu, P. Maksimovic, S. Rappoccio, M. Swartz, A. Whitbeck 
The University of Kansas, Lawrence, USA

P. Baringer, A. Bean, G. Benelli, R.P. Kenny Iii, M. Murray, D. Noonan, S. Sanders, R. Stringer, G. Tinti, J.S. Wood, V. Zhukova

Kansas State University, Manhattan, USA

A.F. Barfuss, T. Bolton, I. Chakaberia, A. Ivanov, S. Khalil, M. Makouski, Y. Maravin, S. Shrestha, I. Svintradze

Lawrence Livermore National Laboratory, Livermore, USA

J. Gronberg, D. Lange, D. Wright

University of Maryland, College Park, USA

A. Baden, M. Boutemeur, B. Calvert, S.C. Eno, J.A. Gomez, N.J. Hadley, R.G. Kellogg, M. Kirn, T. Kolberg, Y. Lu, M. Marionneau, A.C. Mignerey, K. Pedro, A. Peterman, A. Skuja, J. Temple, M.B. Tonjes, S.C. Tonwar, E. Twedt

Massachusetts Institute of Technology, Cambridge, USA

A. Apyan, G. Bauer, J. Bendavid, W. Busza, E. Butz, I.A. Cali, M. Chan, V. Dutta, G. Gomez Ceballos, M. Goncharov, K.A. Hahn, Y. Kim, M. Klute, K. Krajczar ${ }^{57}$, P.D. Luckey, T. Ma, S. Nahn, C. Paus, D. Ralph, C. Roland, G. Roland, M. Rudolph, G.S.F. Stephans, F. Stöckli, K. Sumorok, K. Sung, D. Velicanu, E.A. Wenger, R. Wolf, B. Wyslouch, M. Yang, Y. Yilmaz, A.S. Yoon, M. Zanetti

\section{University of Minnesota, Minneapolis, USA}

S.I. Cooper, B. Dahmes, A. De Benedetti, G. Franzoni, A. Gude, S.C. Kao, K. Klapoetke, Y. Kubota, J. Mans, N. Pastika, R. Rusack, M. Sasseville, A. Singovsky, N. Tambe, J. Turkewitz

\section{University of Mississippi, Oxford, USA}

L.M. Cremaldi, R. Kroeger, L. Perera, R. Rahmat, D.A. Sanders

University of Nebraska-Lincoln, Lincoln, USA

E. Avdeeva, K. Bloom, S. Bose, J. Butt, D.R. Claes, A. Dominguez, M. Eads, J. Keller, I. Kravchenko, J. Lazo-Flores, H. Malbouisson, S. Malik, G.R. Snow

State University of New York at Buffalo, Buffalo, USA

A. Godshalk, I. Iashvili, S. Jain, A. Kharchilava, A. Kumar

Northeastern University, Boston, USA

G. Alverson, E. Barberis, D. Baumgartel, M. Chasco, J. Haley, D. Nash, D. Trocino, D. Wood, J. Zhang

\section{Northwestern University, Evanston, USA}

A. Anastassov, A. Kubik, N. Mucia, N. Odell, R.A. Ofierzynski, B. Pollack, A. Pozdnyakov, M. Schmitt, S. Stoynev, M. Velasco, S. Won 


\section{University of Notre Dame, Notre Dame, USA}

L. Antonelli, D. Berry, A. Brinkerhoff, K.M. Chan, M. Hildreth, C. Jessop, D.J. Karmgard, J. Kolb, K. Lannon, W. Luo, S. Lynch, N. Marinelli, D.M. Morse, T. Pearson, M. Planer, R. Ruchti, J. Slaunwhite, N. Valls, M. Wayne, M. Wolf

\section{The Ohio State University, Columbus, USA}

B. Bylsma, L.S. Durkin, C. Hill, R. Hughes, K. Kotov, T.Y. Ling, D. Puigh, M. Rodenburg, C. Vuosalo, G. Williams, B.L. Winer

\section{Princeton University, Princeton, USA}

N. Adam, E. Berry, P. Elmer, D. Gerbaudo, V. Halyo, P. Hebda, J. Hegeman, A. Hunt, P. Jindal, D. Lopes Pegna, P. Lujan, D. Marlow, T. Medvedeva, M. Mooney, J. Olsen, P. Piroué, X. Quan, A. Raval, B. Safdi, H. Saka, D. Stickland, C. Tully, J.S. Werner, A. Zuranski

\section{University of Puerto Rico, Mayaguez, USA}

E. Brownson, A. Lopez, H. Mendez, J.E. Ramirez Vargas

\section{Purdue University, West Lafayette, USA}

E. Alagoz, V.E. Barnes, D. Benedetti, G. Bolla, D. Bortoletto, M. De Mattia, A. Everett, Z. Hu, M. Jones, O. Koybasi, M. Kress, A.T. Laasanen, N. Leonardo, V. Maroussov, P. Merkel, D.H. Miller, N. Neumeister, I. Shipsey, D. Silvers, A. Svyatkovskiy, M. Vidal Marono, H.D. Yoo, J. Zablocki, Y. Zheng

Purdue University Calumet, Hammond, USA

S. Guragain, N. Parashar

\section{Rice University, Houston, USA}

A. Adair, C. Boulahouache, K.M. Ecklund, F.J.M. Geurts, W. Li, B.P. Padley, R. Redjimi, J. Roberts, J. Zabel

\section{University of Rochester, Rochester, USA}

B. Betchart, A. Bodek, Y.S. Chung, R. Covarelli, P. de Barbaro, R. Demina, Y. Eshaq, T. Ferbel, A. Garcia-Bellido, P. Goldenzweig, J. Han, A. Harel, D.C. Miner, D. Vishnevskiy, M. Zielinski

\section{The Rockefeller University, New York, USA}

A. Bhatti, R. Ciesielski, L. Demortier, K. Goulianos, G. Lungu, S. Malik, C. Mesropian

Rutgers, the State University of New Jersey, Piscataway, USA

S. Arora, A. Barker, J.P. Chou, C. Contreras-Campana, E. Contreras-Campana, D. Duggan, D. Ferencek, Y. Gershtein, R. Gray, E. Halkiadakis, D. Hidas, A. Lath, S. Panwalkar, M. Park, R. Patel, V. Rekovic, J. Robles, K. Rose, S. Salur, S. Schnetzer, C. Seitz, S. Somalwar, R. Stone, S. Thomas

\section{University of Tennessee, Knoxville, USA}

G. Cerizza, M. Hollingsworth, S. Spanier, Z.C. Yang, A. York 


\section{Texas A\&M University, College Station, USA}

R. Eusebi, W. Flanagan, J. Gilmore, T. Kamon ${ }^{58}$, V. Khotilovich, R. Montalvo, I. Osipenkov, Y. Pakhotin, A. Perloff, J. Roe, A. Safonov, T. Sakuma, S. Sengupta, I. Suarez, A. Tatarinov, D. Toback

\section{Texas Tech University, Lubbock, USA}

N. Akchurin, J. Damgov, C. Dragoiu, P.R. Dudero, C. Jeong, K. Kovitanggoon, S.W. Lee, T. Libeiro, Y. Roh, I. Volobouev

\section{Vanderbilt University, Nashville, USA}

E. Appelt, A.G. Delannoy, C. Florez, S. Greene, A. Gurrola, W. Johns, P. Kurt, C. Maguire, A. Melo, M. Sharma, P. Sheldon, B. Snook, S. Tuo, J. Velkovska

\section{University of Virginia, Charlottesville, USA}

M.W. Arenton, M. Balazs, S. Boutle, B. Cox, B. Francis, J. Goodell, R. Hirosky, A. Ledovskoy, C. Lin, C. Neu, J. Wood

\section{Wayne State University, Detroit, USA}

S. Gollapinni, R. Harr, P.E. Karchin, C. Kottachchi Kankanamge Don, P. Lamichhane, A. Sakharov

\section{University of Wisconsin, Madison, USA}

M. Anderson, D. Belknap, L. Borrello, D. Carlsmith, M. Cepeda, S. Dasu, E. Friis, L. Gray, K.S. Grogg, M. Grothe, R. Hall-Wilton, M. Herndon, A. Hervé, P. Klabbers, J. Klukas, A. Lanaro, C. Lazaridis, J. Leonard, R. Loveless, A. Mohapatra, I. Ojalvo, F. Palmonari, G.A. Pierro, I. Ross, A. Savin, W.H. Smith, J. Swanson

\footnotetext{
$\dagger$ : Deceased

1: Also at Vienna University of Technology, Vienna, Austria

2: Also at National Institute of Chemical Physics and Biophysics, Tallinn, Estonia

3: Also at Universidade Federal do ABC, Santo Andre, Brazil

4: Also at California Institute of Technology, Pasadena, USA

5: Also at CERN, European Organization for Nuclear Research, Geneva, Switzerland

6: Also at Laboratoire Leprince-Ringuet, Ecole Polytechnique, IN2P3-CNRS, Palaiseau, France

7: Also at Suez Canal University, Suez, Egypt

8: Also at Zewail City of Science and Technology, Zewail, Egypt

9: Also at Cairo University, Cairo, Egypt

10: Also at Fayoum University, El-Fayoum, Egypt

11: Also at British University, Cairo, Egypt

12: Now at Ain Shams University, Cairo, Egypt

13: Also at National Centre for Nuclear Research, Swierk, Poland

14: Also at Université de Haute-Alsace, Mulhouse, France

15: Now at Joint Institute for Nuclear Research, Dubna, Russia

16: Also at Moscow State University, Moscow, Russia

17: Also at Brandenburg University of Technology, Cottbus, Germany

18: Also at Institute of Nuclear Research ATOMKI, Debrecen, Hungary

19: Also at Eötvös Loránd University, Budapest, Hungary
} 
20: Also at Tata Institute of Fundamental Research - HECR, Mumbai, India

21: Also at University of Visva-Bharati, Santiniketan, India

22: Also at Sharif University of Technology, Tehran, Iran

23: Also at Isfahan University of Technology, Isfahan, Iran

24: Also at Plasma Physics Research Center, Science and Research Branch, Islamic Azad University, Tehran, Iran

25: Also at Facoltà Ingegneria Università di Roma, Roma, Italy

26: Also at Università della Basilicata, Potenza, Italy

27: Also at Università degli Studi Guglielmo Marconi, Roma, Italy

28: Also at Università degli Studi di Siena, Siena, Italy

29: Also at University of Bucharest, Faculty of Physics, Bucuresti-Magurele, Romania

30: Also at Faculty of Physics of University of Belgrade, Belgrade, Serbia

31: Also at University of California, Los Angeles, Los Angeles, USA

32: Also at Scuola Normale e Sezione dell' INFN, Pisa, Italy

33: Also at INFN Sezione di Roma; Università di Roma, Roma, Italy

34: Also at University of Athens, Athens, Greece

35: Also at Rutherford Appleton Laboratory, Didcot, United Kingdom

36: Also at The University of Kansas, Lawrence, USA

37: Also at Paul Scherrer Institut, Villigen, Switzerland

38: Also at Institute for Theoretical and Experimental Physics, Moscow, Russia

39: Also at Gaziosmanpasa University, Tokat, Turkey

40: Also at Adiyaman University, Adiyaman, Turkey

41: Also at Izmir Institute of Technology, Izmir, Turkey

42: Also at The University of Iowa, Iowa City, USA

43: Also at Mersin University, Mersin, Turkey

44: Also at Ozyegin University, Istanbul, Turkey

45: Also at Kafkas University, Kars, Turkey

46: Also at Suleyman Demirel University, Isparta, Turkey

47: Also at Ege University, Izmir, Turkey

48: Also at School of Physics and Astronomy, University of Southampton, Southampton, United Kingdom

49: Also at INFN Sezione di Perugia; Università di Perugia, Perugia, Italy

50: Also at University of Sydney, Sydney, Australia

51: Also at Utah Valley University, Orem, USA

52: Also at Institute for Nuclear Research, Moscow, Russia

53: Also at University of Belgrade, Faculty of Physics and Vinca Institute of Nuclear Sciences, Belgrade, Serbia

54: Also at Argonne National Laboratory, Argonne, USA

55: Also at Erzincan University, Erzincan, Turkey

56: Also at Mimar Sinan University, Istanbul, Istanbul, Turkey

57: Also at KFKI Research Institute for Particle and Nuclear Physics, Budapest, Hungary

58: Also at Kyungpook National University, Daegu, Korea 